Article

\title{
Nonleptonic Decays of Doubly Charmed Baryons
}

\author{
Mikhail A. Ivanov \\ Bogoliubov Laboratory of Theoretical Physics, Joint Institute for Nuclear Research, Dubna 141980, Russia; \\ ivanovm@theor.jinr.ru
}

Received: 6 January 2020; Accepted: 5 February 2020; Published: 19 February 2020

\begin{abstract}
In this lecture, we provide a basic introduction into the topic of charmed baryons and their nonleptonic two-body decays. Some features of the baryon weak decays on the quark level are discussed in detail in the framework of effective field theory. The calculation of the matrix elements of the four-quark operators arising in the effective theory proceeds by using the covariant constituent quark model. The model allows one to evaluate not only the factorizing tree-level diagrams but also more complicated diagrams with the internal $W$-exchange. The technique required for such calculation is discussed in some detail. Finally, the numerical results are presented, and comparison of the contributions coming from the $W$-exchange diagrams with those from the tree-level are carefully performed.
\end{abstract}

Keywords: doubly charmed baryons; covariant constituent quark model; nonleptonic decays

\section{Introduction}

In 1964, Gell-Mann proposed [1] the theory of quarks-fundamental particles that make up most ordinary matter. It was done by using the observation that the successful eightfold way of hadron classification would be naturally explained if hadrons were composed of a quark-antiquark pair or three quarks (or three antiquarks).

In the same year, George Zweig came to the same conclusion independently [2,3] by analyzing the suppressed strong decays of the $\phi$-meson. He called three constituents by aces.

The existence of a fourth quark was discussed by a number of authors around 1964, for instance, by James Bjorken and Sheldon Glashow [4]. But there was little evidence for its existence. Its prediction is usually credited to Glashow-Iliopoulos-Maiani [5] for the so-called GIM mechanism, which forbids the flavor-changing neutral currents in the tree diagrams. It explains why weak interactions that change strangeness by a factor of 2 are suppressed. The first particle containing the charmed quark and antiquark was discovered in 1974 and named as the $J / \psi$ meson.

The masses of singly charmed baryons was predicted in one gluon exchange model developed in Reference [6]. The comprehensive review on heavy baryons, their spectroscopy, and semileptonic and nonleptonic decays may be found in Reference [7]. In Tables 1 and 2, we display the names and quark contents of the low-lying multiplets of charmed baryons with spin $1 / 2$ and $3 / 2$, respectively. The values of masses with errors are taken from PDG [8], whereas without errors from Reference [7]. 
Table 1. Charmed $1 / 2^{+}$baryon states. Notation $[a, b]$ and $\{a, b\}$ for antisymmetric and symmetric flavor index combinations. The third column shows which SU(3) adjoint $\left({ }^{*}\right)$ or fundamental representation the quark state belongs to.

\begin{tabular}{lllll}
\hline Title & Quark Content & $S \boldsymbol{S}(\mathbf{3})$ & $\left(\boldsymbol{I}, \boldsymbol{I}_{\mathbf{3}}\right)$ & Mass $(\mathbf{M e V})$ \\
\hline$\Lambda_{c}^{+}$ & $c[u d]$ & $3^{*}$ & $(0,0)$ & $2286.46 \pm 0.14$ \\
$\mathrm{Z}^{\prime} \Xi_{c}^{+}$ & $c[u s]$ & $3^{*}$ & $(1 / 2,1 / 2)$ & $2467.93 \pm 0.18$ \\
$\Xi_{c}^{0}$ & $c[d s]$ & $3^{*}$ & $(1 / 2,-1 / 2)$ & $2470.91 \pm 0.25$ \\
\hline$\Sigma_{c}^{++}$ & $c u u$ & 6 & $(1,1)$ & $2453.97 \pm 0.14$ \\
$\Sigma_{c}^{+}$ & $c\{u d\}$ & 6 & $(1,0)$ & $2452.9 \pm 0.4$ \\
$\Sigma_{c}^{0}$ & $c d d$ & 6 & $(1,-1)$ & $2453.75 \pm 0.14$ \\
$\Xi_{c}^{\prime+}$ & $c\{u s\}$ & 6 & $(1 / 2,1 / 2)$ & $2578.4 \pm 0.5$ \\
$\Xi_{c}^{\prime 0}$ & $c\{d s\}$ & 6 & $(1 / 2,-1 / 2)$ & $2579.2 \pm 0.5$ \\
$\Omega_{c}^{0}$ & $c s s$ & 6 & $(0,0)$ & $2695.2 \pm 1.7$ \\
\hline$\Xi_{c c}^{++}$ & $u c c$ & 3 & $(1 / 2,1 / 2)$ & $3621.2 \pm 0.7$ \\
$\Xi_{c c}^{+}$ & $d c c$ & 3 & $(1 / 2,-1 / 2)$ & 3610 \\
$\Omega_{c c}^{+}$ & $s c c$ & 3 & $(0,0)$ & 3710 \\
\hline
\end{tabular}

Table 2. Charmed $3 / 2^{+}$baryon states.

\begin{tabular}{lllll}
\hline Title & Quark Content & $\operatorname{SU}(\mathbf{3})$ & $\left(\boldsymbol{I}, \boldsymbol{I}_{\mathbf{3}}\right)$ & Mass $\mathbf{( M e V )}$ \\
\hline$\Sigma_{c}^{*++}$ & $c u u$ & 6 & $(1,1)$ & $2518.41 \pm 0.20$ \\
$\Sigma_{c}^{*+}$ & $c u d$ & 6 & $(1,0)$ & $2517.5 \pm 2.3$ \\
$\Sigma_{c}^{* 0}$ & $c d d$ & 6 & $(1,-1)$ & $2518.48 \pm 0.20$ \\
$\Xi_{c}^{*+}$ & $c u s$ & 6 & $(1 / 2,1 / 2)$ & $2645.57 \pm 0.26$ \\
$\Xi_{c}^{* 0}$ & $c d s$ & 6 & $(1 / 2,-1 / 2)$ & $2646.38 \pm 0.21$ \\
$\Omega_{c}^{* 0}$ & $c s s$ & 6 & $(0,0)$ & $2765.9 \pm 2.0$ \\
\hline$\Xi_{c c}^{*++}$ & $u c c$ & 3 & $(1 / 2,1 / 2)$ & 3680 \\
$\Xi_{c c}^{*+}$ & $d c c$ & 3 & $(1 / 2,-1 / 2)$ & 3680 \\
$\Omega_{c c}^{*+}$ & $s c c$ & 3 & $(0,0)$ & 3760 \\
\hline$\Omega_{c c c}^{*++}$ & $c c c$ & 1 & $(0,0)$ & 4730 \\
\hline
\end{tabular}

The lowest lying multiplet of charmed baryons with spin $1 / 2$ can decay only weakly. Therefore, the study of the nonleptonic decays of charmed baryons is very important in the phenomenology of particle interactions. There are now more precise results on the branching ratios of the two-body decays of charmed baryons $\Lambda_{c}^{+} \rightarrow p \phi, \Lambda \pi^{+}, \Sigma^{+} \pi^{0}$ [9] and $\Xi_{c}^{+} \rightarrow p \bar{K}^{*}(892)^{\circ}$ [10,11]. In 2005, a new era began in the studies of doubly charmed baryons when the SELEX Collaboration reported on the observation of a state with the quantum numbers of the spin $1 / 2$ ground state $\Xi_{c c}^{+}$baryon with a mass of $3518 \pm 3 \mathrm{MeV}$ [12]. The SELEX (Segmented Large X baryon Spectrometer) is a fixed target experiment at Fermilab. This doubly charmed baryon state was conjectured to be an isospin- $\frac{1}{2}$ baryon with quark content $(d c c)$ and to have an isospin partner $\Xi_{c c}^{++}$with the quark structure $(u c c)$. However, other Collaborations (BABAR, Belle, LHCb [8]) found no evidence for the $\Xi_{c c}^{+}$nor the $\Xi_{c c}^{++}$ states in the conjectured mass region of $\sim 3500 \mathrm{MeV}$. Recently, the LHCb Collaboration discovered the doubly charmed state $\Xi_{c c}^{++}[13-15]$ in the invariant mass spectrum of the final state particles $\left(\Lambda_{c}^{+} K^{-} \pi^{+} \pi^{+}\right)$. The LHCb is Large Hadron Collider beauty experiment at CERN. The extracted mass of the $\Xi_{c c}^{++}$state was given as $3621.40 \pm 0.72 \pm 0.27 \pm 0.14 \mathrm{MeV}$ and was $\sim 100 \mathrm{MeV}$ heavier than the mass of the original SELEX doubly charmed baryon state $\Xi_{c c}^{+}$, which made it quite unlikely that the two states were isospin partners. On the other hand, the $\mathrm{LHCb}$ mass measurement was in agreement with theoretical mass value predictions for the doubly charmed baryon states. In particular, the central mass value of the $\mathrm{LHCb}$ result for the $\Xi_{c c}^{++}$was very close the value $3610 \mathrm{MeV}$ and 3620 $\mathrm{MeV}$ predicted in Reference $[7,16]$ using the one gluon exchange model of de Rujula, Georgi, and Glashow [17] and a relativistic quark-diquark potential model [18], respectively. Fleck and Richard, 
using a variety of models, also predicted a mass value of $\sim 3600$ [19], while Karliner et al. found $M_{\Xi_{c c}}=3627 \pm 12 \mathrm{MeV}[20]$.

The new measurement of the LHCb Collaboration has stimulated much theoretical activity of the nonleptonic decays of doubly heavy baryons. The recent review can be found in Reference [21,22].

\section{Light Baryons in SU(3)}

In this section, let us recall the basic features of the $S U(3)$-classification of light baryons. Baryons are classified into multiplets according to decomposition of reducible product of the fundamental quark representations:

$$
3 \otimes 3 \otimes 3=10 \oplus 8 \oplus 8^{\prime} \oplus 1 .
$$

The octet representation 8 is written as (see Reference [23]):

$$
B_{j}^{i}=\frac{1}{2}\left(T^{i} q_{j}-\frac{1}{3} \delta_{j}^{i} T^{k} q_{k}\right)
$$

where a vector $T^{i}$ belonging to the representation $\overline{3}$ can be written as

$$
T^{i}=\frac{1}{\sqrt{2}} \varepsilon^{i j k}\left(q_{j} q_{k}-q_{k} q_{j}\right), \quad \text { with quark triplet } q=\left(\begin{array}{c}
q_{1} \\
q_{2} \\
q_{3}
\end{array}\right)=\left(\begin{array}{c}
u \\
d \\
s
\end{array}\right) .
$$

The matrix form of the octet representation looks like

$$
B_{j}^{i}=\left(\begin{array}{ccc}
\frac{1}{\sqrt{6}} \Lambda^{0}+\frac{1}{\sqrt{2}} \Sigma^{0} & \Sigma^{+} & p \\
\Sigma^{-} & \frac{1}{\sqrt{6}} \Lambda^{0}-\frac{1}{\sqrt{2}} \Sigma^{0} & n \\
\Xi^{-} & \Xi^{0} & -\frac{2}{\sqrt{6}} \Lambda^{0}
\end{array}\right) .
$$

The quark content of baryons are shown in Table 3.

Table 3. Quark content of the baryon octet.

$$
\begin{array}{ccc}
p \rightarrow \frac{1}{\sqrt{2}}[u, d] u & n \rightarrow \frac{1}{\sqrt{2}}[u, d] d & \\
\Sigma^{+} \rightarrow \frac{1}{\sqrt{2}}[s, u] u & \Sigma^{0} \rightarrow \frac{1}{2}([d, s] u+[u, s] d) & \Sigma^{-} \rightarrow \frac{1}{\sqrt{2}}[d, s] d \\
\Lambda^{0} \rightarrow \frac{1}{\sqrt{12}}(-2[u, d] s+[d, s] u+[s, u] d) & \Xi^{-} \rightarrow \frac{1}{\sqrt{2}}[d, s] s & \Xi^{0} \rightarrow \frac{1}{\sqrt{2}}[s, u] s \\
\hline
\end{array}
$$

Up to now, we discussed only the flavor structure of quark fields. Generally speaking, they also depend on color, spin, and space coordinates. Therefore, one has to construct the relativistic three quark currents with quantum numbers corresponding to certain member of the baryon octet. One can start from construction of $q q q$-currents, which are symmetric under permutation of all quarks in the case of exact $S U_{F}(3)$-symmetry. This program has been realized in Reference [24]. The starting point is the relativistic three-quark current with quantum numbers of a baryon octet $J^{P}=\frac{1}{2}^{+}$. The general form of this current can be written as

$$
B_{j}^{k} \rightarrow R_{j}^{k ; j_{1}, j_{2}, j_{3}} q_{j_{1}}^{a_{1}} q_{j_{2}}^{a_{2}} q_{j_{3}}^{a_{3}} \varepsilon_{a_{1}, a_{2}, a_{3}}
$$


where $j=(\alpha, m) ; a_{i}, \alpha_{i}, m_{i}$ are the color, spinor, and flavor indices. By using the Fierz transformations for both Dirac matrices and SU(3)-matrices, one finds that there exist two independent currents for a baryon octet with quantum numbers $J^{P}=\frac{1}{2}^{+}$. They may be written in the form:

$$
J^{k m}=\varepsilon^{k m_{2} m_{1}} \delta^{m m_{3}} \Gamma_{1} q_{m_{1}}^{a_{1}}\left(q_{m_{2}}^{a_{2}} C \Gamma_{2} q_{m_{3}}^{a_{3}}\right) \varepsilon_{a_{1} a_{2} a_{3}}
$$

where $\Gamma_{1} \otimes \Gamma_{2}=\gamma_{\mu} \gamma_{5} \otimes \gamma^{\mu}$ (vector current) or $\sigma_{\mu v} \gamma_{5} \otimes \sigma^{\mu \nu}$ (tensor current). $C=\gamma^{0} \gamma^{2}$ is the usual charge conjugation matrix:

$$
\begin{aligned}
C^{T} & =-C, \quad C^{-1}=C, \quad C^{\dagger}=C . \\
C \Gamma^{T} C^{-1} & =\left\{\begin{array}{cc}
+\Gamma & S, P, A \\
-\Gamma & V, T .
\end{array}\right.
\end{aligned}
$$

One can check that the diquark with identical flavors exists for $\Gamma_{2}=\gamma^{\mu}$ and $\sigma^{\mu v}$ only:

$$
\begin{aligned}
\left(u^{a_{2}} C \Gamma_{2} u^{a_{3}}\right) \varepsilon_{a_{1} a_{2} a_{3}} & =-\left(u^{a_{3}}\left(C \Gamma_{2}\right)^{T} u^{a_{2}}\right) \varepsilon_{a_{1} a_{2} a_{3}} \\
& =-(u^{a_{3}} C \underbrace{C C^{-1} \Gamma_{2}^{T} C^{T}}_{+\Gamma_{2}} u^{a_{2}}) \varepsilon_{a_{1} a_{2} a_{3}}=+\left(u^{a_{2}} C \Gamma_{2} u^{a_{3}}\right) \varepsilon_{a_{1} a_{2} a_{3}} .
\end{aligned}
$$

Finally, the isotopic components for three-quark currents are written as in Table 4. The obtained expressions are coincided with those from Reference [25,26].

Table 4. Three-quark currents of the baryon octet (tensor Levi-Civita $\varepsilon_{a_{1} a_{2} a_{3}}$ skipped).

\begin{tabular}{|c|c|c|c|}
\hline Scalar & $S$ & $I$ & 1 \\
\hline Vector & $\mathrm{V}$ & $\gamma^{\mu}$ & 4 \\
\hline Tensor & $\mathrm{T}$ & $\sigma^{\mu v}=\frac{i}{2}\left[\gamma^{\mu}, \gamma^{v}\right] \quad(\mu<v)$ & 6 \\
\hline Pseudoscalar & $\mathrm{P}$ & $\gamma_{5}=i \gamma^{0} \gamma^{1} \gamma^{2} \gamma^{3}$ & 1 \\
\hline Axial & A & $i \gamma^{\mu} \gamma_{5}$ & 4 \\
\hline
\end{tabular}

$$
\begin{array}{ccc}
p \rightarrow \Gamma_{1} d_{a_{1}}\left(u_{a_{2}} C \Gamma_{2} u_{a_{3}}\right) & n \rightarrow \Gamma_{1} u_{a_{1}}\left(d_{a_{2}} C \Gamma_{2} d_{a_{3}}\right) & \\
\Sigma^{+} \rightarrow \Gamma_{1} s_{a_{1}}\left(u_{a_{2}} C \Gamma_{2} u_{a_{3}}\right) & \Sigma^{0} \rightarrow \sqrt{2} \Gamma_{1} s_{a_{1}}\left(u_{a_{2}} C \Gamma_{2} d_{a_{3}}\right) & \Sigma^{-} \rightarrow \Gamma_{1} s_{a_{1}}\left(d_{a_{2}} C \Gamma_{2} d_{a_{3}}\right) \\
\Lambda^{0} \rightarrow \sqrt{\frac{2}{3}}\left\{\Gamma_{1} u_{a_{1}}\left(d_{a_{2}} C \Gamma_{2} s_{a_{3}}\right)-\Gamma_{1} d_{a_{1}}\left(u_{a_{2}} C \Gamma_{2} s_{a_{3}}\right)\right\} & \Xi^{-} \rightarrow \Gamma_{1} d_{a_{1}}\left(s_{a_{2}} C \Gamma_{2} s_{a_{3}}\right) & \Xi^{0} \rightarrow \Gamma_{1} u_{a_{1}}\left(s_{a_{2}} C \Gamma_{2} s_{a_{3}}\right) \\
\hline
\end{array}
$$

\subsection{Fierz Transformations for Dirac Matrices}

The basis of sixteen $4 \times 4$ Dirac matrices is shown in Table 5 .

Table 5. Basis of Dirac matrices.

The matrices from the basis satisfy to the normalization condition given by Equation (7) in general form and conditions given by Equation (8) for each matrix from the basis.

$$
\operatorname{tr}\left(\Gamma^{C} \Gamma^{D}\right)=4 \delta_{C D} \quad \text { where } \quad(C, D=S, V, T, P, A),
$$




$$
\begin{aligned}
& \operatorname{tr}\left(I_{4} I_{4}\right)=4, \quad \operatorname{tr}\left(\gamma^{\mu} \gamma^{\nu}\right)=4 g^{\mu \nu}, \\
& \operatorname{tr}\left(\gamma_{5} \gamma_{5}\right)=4, \quad \operatorname{tr}\left(i \gamma_{5} \gamma^{\mu} \cdot i \gamma_{5} \gamma^{\nu}\right)=4 g^{\mu \nu}, \\
& \operatorname{tr}\left(\sigma^{\mu v} \sigma^{\alpha \beta}\right)=4\left(g^{\mu \alpha} g^{\nu \beta}-g^{\mu \beta} g^{v \alpha}\right) .
\end{aligned}
$$

Any product of Dirac matrices can be decomposed into 16 independent matrices:

$$
\Gamma=\sum_{D=S, V, T, P, A} C^{D} \Gamma^{D}, \quad \text { where } 4 C^{D}=\operatorname{tr}\left(\Gamma \Gamma^{D}\right) .
$$

One can derive from Equation (9) the useful property of the Dirac matrices called the Fierz transformation. One has

$$
4 \Gamma_{\alpha_{1} \alpha_{2}}=\sum_{D} \operatorname{tr}\left(\Gamma \Gamma^{D}\right) \Gamma_{\alpha_{1} \alpha_{2}}^{D}=\Gamma_{\alpha_{4} \alpha_{3}} \sum_{D} \Gamma_{\alpha_{3} \alpha_{4}}^{D} \Gamma_{\alpha_{1} \alpha_{2}}^{D}=4 \Gamma_{\alpha_{1} \alpha_{2}} .
$$

The last equality holds if and only if the following property is valid:

$$
\sum_{D=S, V, T, P, A} \Gamma_{\alpha_{1} \alpha_{2}}^{D} \Gamma_{\alpha_{3} \alpha_{4}}^{D}=4 \delta_{\alpha_{1} \alpha_{4}} \delta_{\alpha_{3} \alpha_{2}} .
$$

By using following, Equation (11), one can arrive at the Fierz transformation

$$
\begin{aligned}
4 \Gamma_{\alpha_{1} \alpha_{2}}^{(1)} \Gamma_{\alpha_{3} \alpha_{4}}^{(2)} & =4 \delta_{\beta_{2} \alpha_{2}} \delta_{\beta_{4} \alpha_{4}} \Gamma_{\alpha_{1} \beta_{2}}^{(1)} \Gamma_{\alpha_{3} \beta_{4}}^{(2)}=\sum_{D} \Gamma_{\beta_{2} \alpha_{4}}^{D} \Gamma_{\beta_{4} \alpha_{2}}^{D} \Gamma_{\alpha_{1} \beta_{2}}^{(1)} \Gamma_{\alpha_{3} \beta_{4}}^{(2)} \\
& =\sum_{D}\left(\Gamma^{(1)} \Gamma^{D}\right)_{\alpha_{1} \alpha_{4}}\left(\Gamma^{(2)} \Gamma^{D}\right)_{\alpha_{3} \alpha_{2}} .
\end{aligned}
$$

Then, we introduce the short notation.

$$
\Gamma_{\alpha_{1} \alpha_{2}}^{(1)} \Gamma_{\alpha_{3} \alpha_{4}}^{(2)}=\widetilde{\Gamma^{(1)}} \otimes \widetilde{\Gamma^{(2)}}, \quad \text { and } \quad \Gamma_{\alpha_{1} \alpha_{4}}^{(1)} \Gamma_{\alpha_{3} \alpha_{2}}^{(2)}=\Gamma^{(1)} \otimes \Gamma^{(2)} .
$$

There are a plenty of useful identities which relate the product of two Dirac matrices with tilde to product of two Dirac matrices without tilde. Here, are some of them:

$$
\begin{aligned}
4 \widetilde{\gamma^{\mu}} \otimes \widetilde{\gamma_{\mu}} & =+4 I \otimes I-2 \gamma^{\mu} \otimes \gamma_{\mu}-2 \gamma^{\mu} \gamma_{5} \otimes \gamma_{\mu} \gamma_{5}-4 \gamma_{5} \otimes \gamma_{5} \\
4 \widetilde{\gamma^{\mu} \gamma_{5}} \otimes \widetilde{\gamma_{\mu} \gamma_{5}} & =-4 I \otimes I-2 \gamma^{\mu} \otimes \gamma_{\mu}-2 \gamma^{\mu} \gamma_{5} \otimes \gamma_{\mu} \gamma_{5}+4 \gamma_{5} \otimes \gamma_{5} \\
4 \widetilde{\gamma^{\mu}} \otimes \widetilde{\gamma_{\mu} \gamma_{5}} & =-4 I \otimes \gamma_{5}+4 \gamma_{5} \otimes I-2 \gamma^{\mu} \otimes \gamma_{\mu} \gamma_{5}-2 \gamma^{\mu} \gamma_{5} \otimes \gamma_{\mu} \\
4 \widetilde{\gamma^{\mu} \gamma_{5}} \otimes \widetilde{\gamma_{\mu}} & =+4 I \otimes \gamma_{5}-4 \gamma_{5} \otimes I-2 \gamma^{\mu} \otimes \gamma_{\mu} \gamma_{5}-2 \gamma^{\mu} \gamma_{5} \otimes \gamma_{\mu} \\
4 \widetilde{I} \otimes \widetilde{\gamma_{5}} & =+I \otimes \gamma_{5}+\gamma_{5} \otimes I-\gamma^{\mu} \otimes \gamma_{\mu} \gamma_{5}+\gamma^{\mu} \gamma_{5} \otimes \gamma_{\mu}+\frac{1}{2} \sigma^{\mu v} \gamma_{5} \otimes \sigma_{\mu \nu} \\
4 \widetilde{\gamma_{5}} \otimes \widetilde{I} & =+I \otimes \gamma_{5}+\gamma_{5} \otimes I+\gamma^{\mu} \otimes \gamma_{\mu} \gamma_{5}-\gamma^{\mu} \gamma_{5} \otimes \gamma_{\mu}+\frac{1}{2} \sigma^{\mu v} \gamma_{5} \otimes \sigma_{\mu v} \\
4 \widetilde{\sigma_{\mu \nu} \gamma_{5}} \otimes \widetilde{\sigma^{\mu \nu}}= & +12 I \otimes \gamma_{5}+12 \gamma_{5} \otimes I-2 \sigma^{\mu v} \gamma_{5} \otimes \sigma_{\mu \nu} .
\end{aligned}
$$

We will use the above identities to simplify the string of Dirac matrices involving two weak matrices with Left/Right chirality $O_{L / R}^{\mu}=\gamma^{\mu}\left(I \mp \gamma_{5}\right)$. One can prove that

$$
\widetilde{O_{L / R}} \otimes \widetilde{O_{L / R}}=-O_{L / R} \otimes O_{L / R}, \quad \widetilde{O_{L / R}} \otimes \widetilde{O_{R / L}}=2\left(I \pm \gamma_{5}\right) \otimes\left(I \mp \gamma_{5}\right) .
$$

By using these properties of the weak matrices, one can get the following simplifications:

$$
\begin{aligned}
\operatorname{tr}\left(\Gamma_{1} O_{L / R} \Gamma_{2} O_{L / R}\right) & =-\operatorname{tr}\left(\Gamma_{1} O_{L / R}\right) \cdot \operatorname{tr}\left(\Gamma_{2} O_{L / R}\right), \\
\left(\Gamma_{1} O_{R} \Gamma_{2} O_{L} \Gamma_{3}\right)^{\alpha \beta} & =2\left(\Gamma_{1}\left(I-\gamma_{5}\right) \Gamma_{3}\right)^{\alpha \beta} \cdot \operatorname{tr}\left[\Gamma_{2}\left(I+\gamma_{5}\right)\right] .
\end{aligned}
$$




\section{2. $S U(n)-M a t r i c e s$}

Here, let us recall some properties of the unitary matrices from the $S U(n)$-group. For $S U(n)$-group, there are $m=n^{2}-1$ generators $t^{a}=\frac{1}{2} \lambda^{a}$, where $\lambda^{a}$ are the Gell-Mann traceless $n \times n$ matrices $\left(a=1, \ldots, m=n^{2}-1\right)$. They satisfy the following relations:

$$
\left[\lambda^{a}, \lambda^{b}\right]=2 i f^{a b c} \lambda^{c}, \quad \operatorname{tr}\left(\lambda^{a} \lambda^{b}\right)=2 \delta^{a b}, \quad(a, b=1, \ldots, m) .
$$

For completeness, one has to add the unit matrix $\lambda^{0}=\sqrt{\frac{2}{n}} I_{n}$. Then, any $n \times n$ matrix can be decomposed into basis matrices:

$$
M=\sum_{a=0}^{m} M^{a} \lambda^{a}, \quad M^{a}=\frac{1}{2} \operatorname{tr}\left(\lambda^{a} M\right)
$$

From Equation (18), it is easy to derive the completeness condition:

$$
\sum_{a=0}^{m} \lambda_{m_{1} m_{2}}^{a} \lambda_{m_{3} m_{4}}^{a}=2 \delta_{m_{1} m_{4}} \delta_{m_{3} m_{2}} .
$$

Some corollaries follow from Equation (19):

$$
\begin{aligned}
& \sum_{a=1}^{m} \lambda_{m_{1} m_{2}}^{a} \lambda_{m_{3} m_{4}}^{a}=2 \delta_{m_{1} m_{4}} \delta_{m_{3} m_{2}}-\frac{2}{n} \delta_{m_{1} m_{2}} \delta_{m_{3} m_{4}} \\
& \sum_{a=1}^{m} \lambda_{m_{1} m_{2}}^{a} \lambda_{m_{3} m_{4}}^{a}=\frac{2\left(n^{2}-1\right)}{n^{2}} \delta_{m_{1} m_{4}} \delta_{m_{3} m_{2}}-\frac{1}{n} \sum_{a=1}^{m} \lambda_{m_{1} m_{4}}^{a} \lambda_{m_{3} m_{2}}^{a} \cdot
\end{aligned}
$$

They allow to simplify the calculation of traces:

$$
\begin{aligned}
& \sum_{a=1}^{m} \operatorname{tr}\left(\lambda^{a} M_{1} \lambda^{a} M_{2}\right)=-\frac{2}{n} \operatorname{tr}\left(M_{1} M_{2}\right)+2 \operatorname{tr}\left(M_{1}\right) \operatorname{tr}\left(M_{2}\right) \\
& \sum_{a=1}^{m} \operatorname{tr}\left(\lambda^{a} M_{1}\right) \operatorname{tr}\left(\lambda^{a} M_{2}\right)=2 \operatorname{tr}\left(M_{1} M_{2}\right)-\frac{2}{n} \operatorname{tr}\left(M_{1}\right) \operatorname{tr}\left(M_{2}\right) .
\end{aligned}
$$

\section{Charmed Baryons}

The existence of a fourth quark had been discussed by a number of authors around 1964, for instance, by James Bjorken and Sheldon Glashow [4]. The addition of the charmed quark to the $(u d s)$ triplet extends the $S U(3)$ to $S U(4)$. The irreducible representations are formed according to decomposition

$$
4 \otimes 4 \otimes 4=20_{S} \oplus 20_{M} \oplus 20_{M}^{\prime} \oplus 4_{A} .
$$

But, in that time, there was little evidence for existence of the charmed quark and related hadrons. Its prediction is usually credited to Glashow-Iliopoulos-Maiani [5] for the so-called GIM mechanism, which forbids the flavor-changing neutral currents in the tree diagrams.

\section{GIM Mechanism}

The attempt to construct the weak interactions with three ( $u d s)$ quarks led to existence of the flavor-changing neutral current (FCNC) at tree level. The left weak duplet is written as

$$
Q_{L}^{u}=\left(\begin{array}{c}
u \\
d \cos \theta_{C}+s \sin \theta_{C}
\end{array}\right)_{L},
$$


where $\theta_{C}$ is the Cabbibo angle. It gives the following contribution to the weak Lagrangian

$$
\begin{aligned}
\bar{Q}_{L}^{u} \gamma^{\mu} \frac{\tau^{3}}{2} Q_{L}^{u}= & \frac{1}{2}\left(\bar{u}_{L} \gamma^{\mu} u_{L}-\cos ^{2} \theta_{C} \bar{d}_{L} \gamma^{\mu} d_{L}-\sin ^{2} \theta_{C} \bar{s}_{L} \gamma^{\mu} S_{L}\right. \\
& \left.-\sin \theta_{C} \cos \theta_{C}\left[\bar{d}_{L} \gamma^{\mu} S_{L}+\bar{s}_{L} \gamma^{\mu} d_{L}\right]\right)
\end{aligned}
$$

The charm quark allows one to construct an extra duplet:

$$
Q_{L}^{c}=\left(\begin{array}{c}
c \\
-d \sin \theta_{C}+s \cos \theta_{C}
\end{array}\right)_{L} .
$$

It will give the additional contribution the the weak Lagrangian, where the FCNC contribute with the opposite sign:

$$
\begin{aligned}
\bar{Q}_{L}^{c} \gamma^{\mu} \frac{\tau^{3}}{2} Q_{L}^{c}= & \frac{1}{2}\left(\bar{c}_{L} \gamma^{\mu} c_{L}-\sin ^{2} \theta_{C} \bar{d}_{L} \gamma^{\mu} d_{L}-\cos ^{2} \theta_{C} \bar{s}_{L} \gamma^{\mu} s_{L}\right. \\
& \left.+\sin \theta_{C} \cos \theta_{C}\left(\bar{d}_{L} \gamma^{\mu} s_{L}+\bar{s}_{L} \gamma^{\mu} d_{L}\right)\right) .
\end{aligned}
$$

As the result, the FCNC vanish from the weak Lagrangian and occur only at the level of loop diagrams:

$$
\bar{Q}_{L}^{u} \gamma^{\mu} \frac{\tau^{3}}{2} Q_{L}^{u}+Q_{L}^{c} \gamma^{\mu} \frac{\tau^{3}}{2} Q_{L}^{c}=\frac{1}{2}\left(\bar{u}_{L} \gamma^{\mu} u_{L}+\bar{c}_{L} \gamma^{\mu} c_{L}-\bar{d}_{L} \gamma^{\mu} d_{L}-\bar{s}_{L} \gamma^{\mu} s_{L}\right)
$$

For instance, the weak decay $K_{L}^{0} \rightarrow \mu^{+} \mu^{-}$goes via sum of one-loop diagrams with u-quark and c-quark, as shown in Figure 1.

The matrix element is proportional to

$$
M\left(K \rightarrow \mu^{+} \mu^{-}\right) \propto \frac{g_{2}^{4} \sin \theta_{c} \cos \theta_{C}}{M_{W}^{2}} \frac{m_{c}^{2}-m_{u}^{2}}{M_{W}^{2}} .
$$

The experimental data can be reproduced if the value of the charm quark mass is of order $\longrightarrow$ $m_{c} \approx 1.5 \mathrm{GeV}$. It was the first solid confirmation of the charm in particle physics.
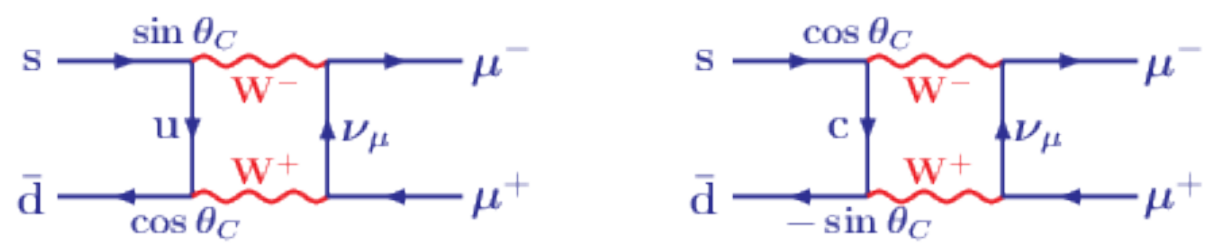

Figure 1. Diagrams describing the decay $K_{L}^{0} \rightarrow \mu^{+} \mu^{-}$. The left diagram describes the exchange by u-quark whereas the right by c-quark.

\section{Nonleptonic Two-Body Weak Decays of Baryons}

Ground states of baryons with $J^{P}=\frac{1}{2}^{+}$can decay only weakly via the internal $W$-exchange. Two-body decays of baryons have five different quark topologies shown in Figure 2. 


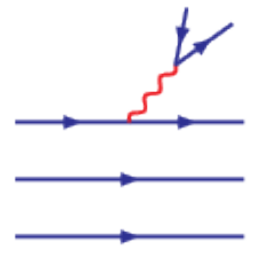

Ia

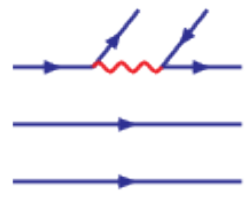

$\mathrm{Ib}$

Tree diagrams

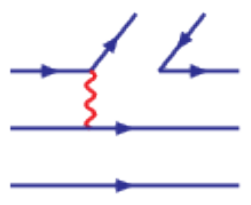

IIa

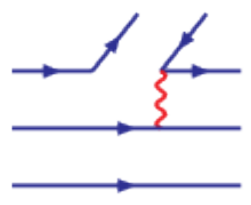

IIb

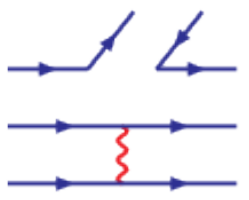

III

\section{W-exchange diagrams}

Figure 2. Quark diagrams with five different topologies. The set of diagrams divides in two groups: (1) The reducible tree-diagrams called external (Ia) and internal W-emission (IIb) diagrams. (2) The irreducible $W$-exchange diagrams with the labeling introduced in $[7,16]$. In [27] the $W$-exchange diagrams are denoted as the Exchange (IIa), color-commensurate (IIb) and Bow tie (III) diagram.

The weak interactions of quarks at energy significantly less than the W-mass, the scale of charm quark mass in the given decays, are described in the framework of an effective low-energy theory. The formal framework is using the Wilson operator product expansion (OPE). As an example, let us consider the tree-level $W$-exchange amplitude for $c \rightarrow s u \bar{d}$ transition. One has

$$
\begin{aligned}
A & =-\frac{g_{2}^{2}}{8} V_{c s}^{*} V_{u d}\left(\bar{s} O^{\mu} c\right)\left(\frac{-g_{\mu v}}{M_{W}^{2}-k^{2}}\right)\left(\bar{u} O^{v} d\right) \\
& =-\frac{g_{2}^{2}}{8 M_{W}^{2}} V_{c s}^{*} V_{u d}\left(\bar{s} O^{\mu} c\right)\left(\bar{u} O_{\mu} d\right)+\mathcal{O}\left(\frac{k^{2}}{M_{W}^{2}}\right),
\end{aligned}
$$

where $O^{\mu}=\gamma^{\mu}\left(1-\gamma_{5}\right)$ is the weak Dirac matrix with left chirality, $V_{c s}$ and $V_{u d}$ are the CKM-matrix elements, and $g_{2}$ is the coupling of gauge group. Since the momentum transfer is less than the $\mathrm{W}$-mass, i.e., $|k|<<M_{W}$, the terms of order $\mathcal{O}\left(k^{2} / M_{W}^{2}\right)$ may be neglected. It is easy to see that the leading term of the expansion can be obtained from an effective Hamiltonian

$$
\mathcal{H}_{\text {eff }}^{\text {tree }}=\frac{G_{F}}{\sqrt{2}} V_{c s}^{*} V_{u d}\left(\bar{s}_{a} O^{\mu} c_{a}\right)\left(\bar{u}_{b} O_{\mu} d_{b}\right), \quad \frac{G_{F}}{\sqrt{2}}=\frac{g_{2}^{2}}{8 M_{W}^{2}} .
$$

Then, one needs to take into account the one-loop QCD corrections both in full theory and in the theory with effective Hamiltonian. The corresponding tree and one-loop diagrams in full and effective theory are shown in Figure 3. 


\section{Current-current diagrams}

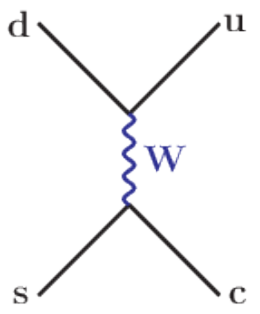

tree

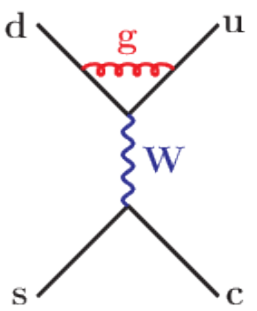

$\mathrm{S}_{\mathrm{C}}$

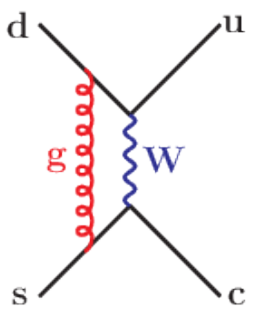

OCD one-loop

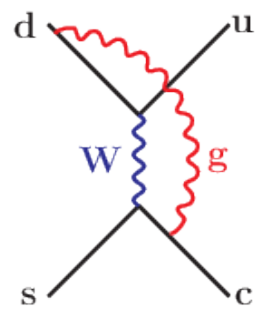

\section{Effective Theory}
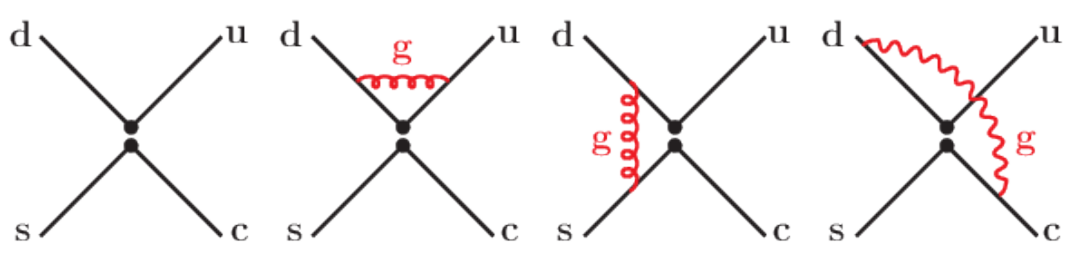

Figure 3. Tree and one-loop diagrams in full and effective theory. In the full theory the tree diagram is described by exchange of the W-boson, whereas in the effective theory the tree diagram is generated by four-quark operator from an effective Hamiltonian. In both theories the next-to-leading corrections are described by gluon (g) exchanges between color quarks.

Including QCD corrections, the effective Hamiltonian is generalized to

$$
\begin{aligned}
& \mathcal{H}_{\mathrm{eff}}=\frac{G_{F}}{\sqrt{2}} V_{c s}^{*} V_{u d}\left(C_{1}(\mu) Q_{1}+C_{2}(\mu) Q_{2}\right), \\
& Q_{1} \equiv\left(\bar{s}_{a} O^{\mu} c_{b}\right)\left(\bar{u}_{b} O_{\mu} d_{a}\right), \quad Q_{2} \equiv\left(\bar{s}_{a} O^{\mu} c_{a}\right)\left(\bar{u}_{b} O_{\mu} d_{b}\right),
\end{aligned}
$$

where the Wilson coefficients $C_{i}(\mu)$ are determined from the matching full and effective theories. The leading order in the strong QCD coupling $\alpha_{S}$ looks as follows:

$$
C_{1}=-3 \frac{\alpha_{S}}{4 \pi} \ln \frac{M_{W}^{2}}{\mu^{2}}, \quad C_{2}=1+\frac{\alpha_{S}}{4 \pi} \ln \frac{M_{W}^{2}}{\mu^{2}} .
$$

Obviously, this expansion will be reliable if the scale $\mu$ is of order of $W$-mass, i.e., $\left.\mu \approx M_{W}\right)$. In this case, the value of $\alpha_{S}$ is small due to asymptotic freedom, and the value of the logarithm is small, as well. Then, the Wilson coefficients are evaluated from $\mu_{W}$ down to the low-energy scale $\mu \propto m_{c}$. The last step is to calculate the hadronic matrix elements of the operators $\left\langle Q_{i}(\mu)\right\rangle$ that requires the nonperturbative methods.

\section{Covariant Constituent Quark Model}

The Covariant Constituent Quark Model (CCQM) is based on a phenomenological, nonlocal, relativistic Lagrangian describing the coupling of a hadron $H$ to its constituents:

$$
\mathcal{L}_{\text {int }}=g_{H} \cdot H(x) \cdot J_{H}(x),
$$


where $J_{H}(x)$ is the quark current corresponding to a hadron $H$. For mesons, baryons, and tetraquarks, the corresponding quark currents may be written as

$$
\begin{aligned}
J_{M}(x)= & \int d x_{1} \int d x_{2} F_{M}\left(x ; x_{1}, x_{2}\right) \cdot \bar{q}_{f_{1}}^{a}\left(x_{1}\right) \Gamma_{M} q_{f_{2}}^{a}\left(x_{2}\right), \\
J_{B}(x)= & \int d x_{1} \int d x_{2} \int d x_{3} F_{B}\left(x ; x_{1}, x_{2}, x_{3}\right) \times \Gamma_{1} q_{f_{1}}^{a_{1}}\left(x_{1}\right)\left(\varepsilon^{a_{1} a_{2} a_{3}} q_{f_{2}}^{T a_{2}}\left(x_{2}\right) C \Gamma_{2} q_{f_{3}}^{a_{3}}\left(x_{3}\right)\right) \\
J_{T}(x)= & \int d x_{1} \ldots \int d x_{4} F_{T}\left(x ; x_{1}, \ldots, x_{4}\right) \\
& \times\left(\varepsilon^{a_{1} a_{2} c} q_{f_{1}}^{T a_{1}}\left(x_{1}\right) C \Gamma_{1} q_{f_{2}}^{a_{2}}\left(x_{2}\right)\right) \cdot\left(\varepsilon^{a_{3} a_{4} c} \bar{q}_{f_{3}}^{T a_{3}}\left(x_{3}\right) \Gamma_{2} C \bar{q}_{f_{4}}^{a_{4}}\left(x_{4}\right)\right) .
\end{aligned}
$$

where $a_{i}$ and $f_{i}$ are color and flavor indices. The vertex functions $F_{H}$ should satisfy to translational invariance

$$
F_{H}\left(x+a ; x_{1}+a, \ldots, x_{n}+a\right)=F_{H}\left(x ; x_{1}, \ldots, x_{n}\right), \quad \forall a .
$$

The simple and obvious choice is written down as

$$
\begin{aligned}
& F_{H}\left(x, x_{1}, \ldots, x_{n}\right)=\delta^{(4)}\left(x-\sum_{i=1}^{n} w_{i} x_{i}\right) \Phi_{H}\left(\sum_{i<j}\left(x_{i}-x_{j}\right)^{2}\right), \\
& \Phi_{H}\left(\sum_{i<j}\left(x_{i}-x_{j}\right)^{2}\right)=\prod_{i=1}^{n-1} \int \frac{d^{4} q_{i}}{(2 \pi)^{4}} e^{-i q_{1}\left(x_{1}-x_{n}\right)-i q_{2}\left(x_{2}-x_{n}\right)-\ldots-i q_{n-1}\left(x_{n-1}-x_{n}\right)} \widetilde{\Phi}_{H}\left(-\frac{1}{2} \sum_{i \leq j} q_{i} q_{j}\right), \\
& \widetilde{\Phi}_{H}(-\Omega) \\
& =\exp \left\{\Omega / \Lambda_{H}^{2}\right\},
\end{aligned}
$$

where $w_{i}=m_{i} / \sum_{j} m_{j}$ so that $\sum_{i=1}^{n} w_{i}=1$, and $\Lambda_{H}$ is an adjustable size parameter. The quark propagators are chosen in the local Dirac form

$$
S_{i}\left(x_{1}-x_{2}\right)=\int \frac{d^{4} k}{(2 \pi)^{4} i} \frac{e^{-i k\left(x_{1}-x_{2}\right)}}{m_{i}-\not k} .
$$

The quark confinement is realized by implementation of the so-called infrared confinement. We discuss how it works in the next sections.

\subsection{Heavy Quark Limit in $B-D\left(D^{*}\right)$ Transition}

It is instructive to explore the heavy quark limit (HQL) in the heavy-to-heavy transition $B \rightarrow$ $D\left(D^{*}\right)$. The diagram describing the semileptonic decay $B \rightarrow D\left(D^{*}\right)+\ell \bar{v}_{\ell}$ is shown in Figure 4. 


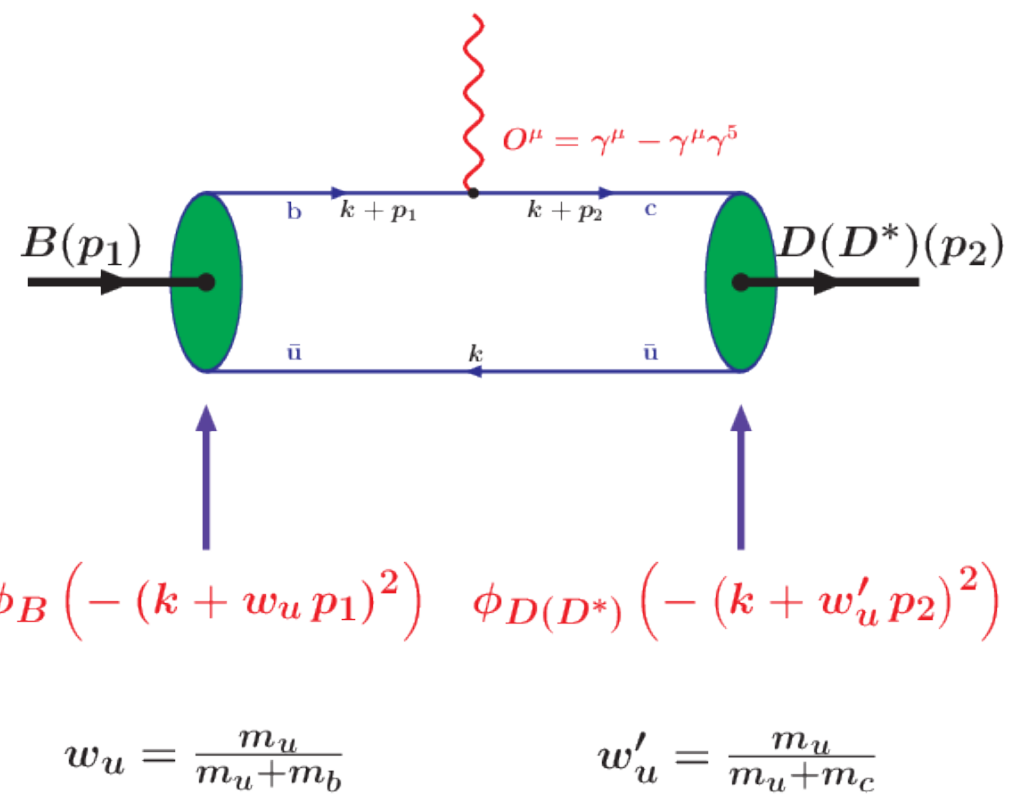

Figure 4. Diagrams describing the semileptonic decay $B \rightarrow D\left(D^{*}\right)+\ell \bar{v}_{\ell}$.

In the HQL, one takes the limit $m_{B}=m_{b}+E, m_{b} \rightarrow \infty$ and $m_{D}=m_{D^{*}}=m_{c}+E, m_{c} \rightarrow \infty$ in the expressions for the coupling constants and form factors. In this limit, the heavy quark propagators are reduced to the static form:

$$
\begin{aligned}
& S_{b}\left(k+p_{1}\right)=\frac{1}{m_{b}-\not k-\not p_{1}} \rightarrow \frac{1+\not b_{1}}{-2 k v_{1}-2 E}+O\left(\frac{1}{m_{b}}\right), \\
& S_{c}\left(k+p_{2}\right)=\frac{1}{m_{c}-\not k-\not p_{2}} \rightarrow \frac{1+\not b_{2}}{-2 k v_{2}-2 E}+O\left(\frac{1}{m_{c}}\right),
\end{aligned}
$$

where $p_{i}$ and $v_{i}=p_{i} / m_{i}(i=1,2)$ are the momenta and the four-velocities of the initial and final states. Moreover, we have to keep the size parameters of heavy hadrons equal to each other in order to provide the correct normalization of the Isgur-Wise function at zero recoil. By using technique developed in our previous papers [28,29], one can arrive at the following expressions for the semileptonic heavy-to-heavy transitions:

$$
\begin{aligned}
T_{\mathrm{HQL}}^{\mu} & =\xi(w) \cdot \frac{1}{4} \operatorname{tr}\left(O^{\mu}\left(1+\not \phi_{1}\right) \gamma^{5} \cdot \gamma^{5}\left(1+\not \not_{2}\right)\right)=\xi(w) \cdot\left(v_{1}^{\mu}+v_{2}^{\mu}\right), \\
\epsilon_{2 v}^{\dagger} T_{\mathrm{HQL}}^{\mu \nu} & =\xi(w) \cdot \frac{1}{4} \operatorname{tr}\left(O^{\mu}\left(1+\not \phi_{1}\right) \gamma^{5} \cdot \notin_{2}^{\dagger}\left(1+\not \phi_{2}\right)\right) \\
& =\xi(w) \cdot \epsilon_{2 v}^{\dagger}\left(-g^{\mu v}(1+w)+v_{1}^{\mu} v_{2}^{v}+v_{1}^{v} v_{2}^{\mu}-i \varepsilon^{\mu v v_{1} v_{2}}\right) .
\end{aligned}
$$

Here, $w=v_{1} v_{2}$, and the Isgur-Wise function is equal to

$$
\xi(w)=\frac{J_{3}(E, w)}{J_{3}(E, 1)}, \quad J_{3}(E, w)=\int_{0}^{1} \frac{d \tau}{W} \int_{0}^{\infty} d u \widetilde{\Phi}^{2}(z) \frac{m_{u}+\sqrt{u / W}}{m_{u}^{2}+z},
$$


where $W=1+2 \tau(1-\tau)(w-1), z=u-2 E \sqrt{u / W}$, and $\widetilde{\Phi}(z)=\exp \left(-z / \Lambda^{2}\right)$. By using the definition of the form factors given in Reference [28,29], one can easily obtain the expressions of the form factors in the HQL. One finds

$$
\begin{aligned}
& F_{ \pm}\left(q^{2}\right)= \pm \frac{m_{1} \pm m_{2}}{2 \sqrt{m_{1} m_{2}}} \xi(w), \\
& A_{0}\left(q^{2}\right)=\frac{\sqrt{m_{1} m_{2}}}{m_{1}-m_{2}}(1+w) \xi(w), \quad A_{+}\left(q^{2}\right)=-A_{-}\left(q^{2}\right)=V\left(q^{2}\right)=\frac{m_{1}+m_{2}}{2 \sqrt{m_{1} m_{2}}} \xi(w),
\end{aligned}
$$

where $w=\left(m_{1}^{2}+m_{2}^{2}-q^{2}\right) /\left(2 m_{1} m_{2}\right)$. We use the physical masses of the heavy hadrons in the numerical calculations. For the size parameter, we adopt the average value $\Lambda=\left(\Lambda_{B}+\Lambda_{D}+\Lambda_{D^{*}}\right) / 3=$ $1.70 \mathrm{GeV}$. The parameter $E$ characterizes the difference in mass between the heavy hadron and the corresponding heavy quark. We use its minimal value $E=m_{D}-m_{c}=0.20 \mathrm{GeV}$ in order to avoid the complication with confinement. In Figure 5, we display the heavy-to-heavy transition form factors calculated in the HQL and compare them with the results of exact calculations [30]. One can see that the two results obtained with and without use of the HQL behave very similar to each other, which demonstrates the fidelity of HQET.

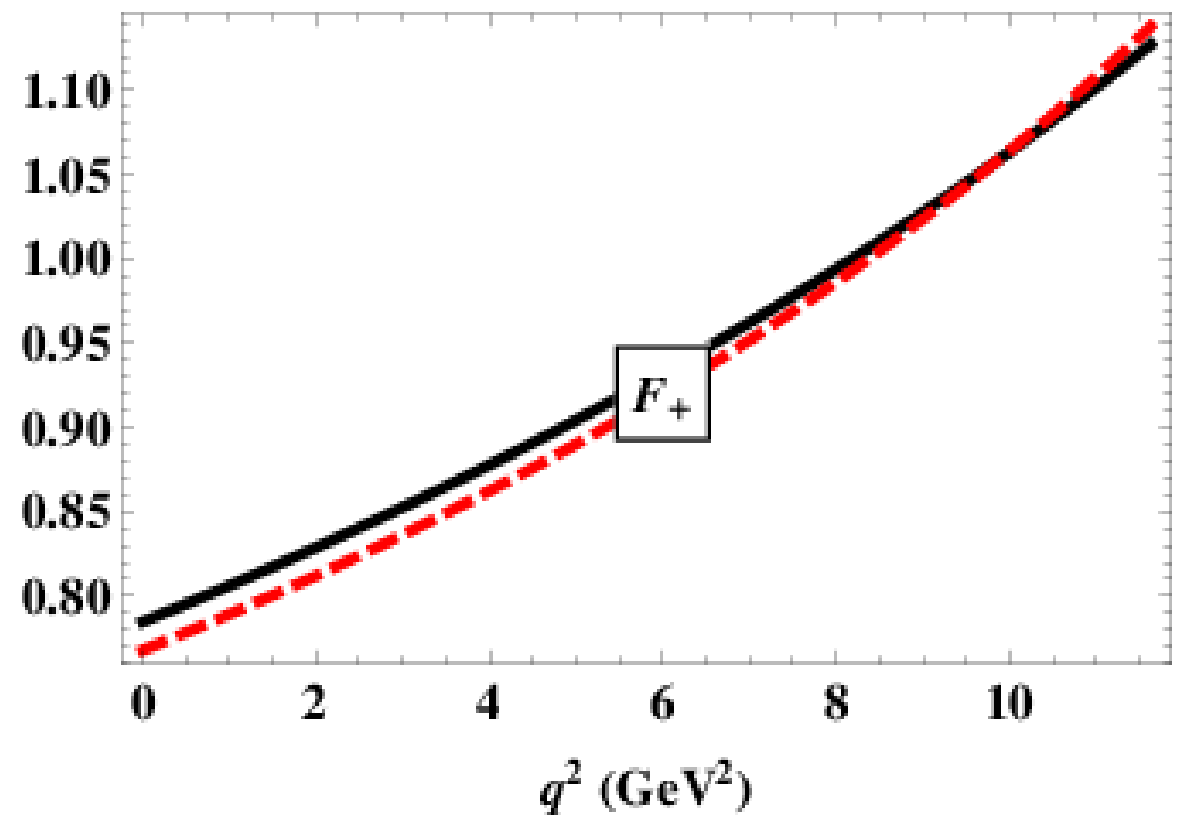

Figure 5. Comparison of the exact calculation of the form factor $F_{+}\left(q^{2}\right)$ (solid line) with those obtained in heavy quark limit (dashed line).

\subsection{Infrared Confinement}

We have shown in Reference [31] how the confinement of quarks can be effectively incorporated in the covariant quark model. In a first step, we introduced an additional scale integration in the space of Fock-Schwinger's $\alpha$-parameters with an integration range from zero to infinity. In a second step, the scale integration was cut off at the upper limit which corresponds to the introduction of an infrared (IR) cutoff. In this manner, all possible thresholds present in the initial quark diagram were removed. The cutoff parameter was taken to be the same for all physical processes. Other model parameters, such as the constituent quark masses and size parameters, were determined from a fit to experimental data.

Let us describe the basic features of how IR confinement is implemented in our model. All physical matrix elements are described by Feynman diagrams written in terms of a convolution of 
free quark propagators and the vertex functions. In computation of Feynman diagrams, we use, in the momentum space, the Fock-Schwinger representation of the quark propagator:

$$
S(k)=\frac{m+\not k}{m^{2}-k^{2}}=(m+\not k) \int_{0}^{\infty} d \alpha e^{-\alpha\left(m^{2}-k^{2}\right)} .
$$

The general form of a resulting Feynman diagrams is

$$
\Pi\left(p_{1}, \ldots, p_{m}\right)=\int_{0}^{\infty} d^{n} \alpha \int\left(d^{4} k\right)^{\ell} \times \operatorname{Num} \times \exp \left\{-\sum_{i=1}^{n} \alpha_{i}\left(m_{i}^{2}-\left(K_{i}+P_{i}\right)^{2}\right)\right\},
$$

where $K_{i}$ represents a linear combination of loop momenta, $P_{i}$ stands for a linear combination of external momenta, and Num refers to the numerator product of propagators and vertex functions. The integrand in Equation (42) has a Gaussian form with the exponential factor

$$
k a k+2 k r+R=k_{i} a_{i j} k_{j}+2 k_{i} r_{i}+R, \quad(i, j=1, \ldots, \ell),
$$

where $k_{i}$ is a 4-vector of the " $\mathrm{i}$ "-loop integration, $a$ is a $\ell \times \ell$ matrix depending on the parameters $\alpha_{i}$ and size parameters $\Lambda, r_{i}$ is a 4- vector composed from the external momenta $p_{i}$, and $R$ is a quadratic form of the masses and external momenta. Tensor loop integrals are calculated with the help of the differential representation

$$
k_{i}^{\mu} e^{2 k r}=\frac{1}{2} \frac{\partial}{\partial r_{i \mu}} e^{2 k r},
$$

which, in general, may be written in the form

$$
\int\left(d^{4} k\right)^{\ell} P(k) e^{k a k+2 k r+R}=\int\left(d^{4} k\right)^{\ell} P\left(\frac{1}{2} \frac{\partial}{\partial r}\right) e^{k a k+2 k r+R}=P\left(\frac{1}{2} \frac{\partial}{\partial r}\right) \int\left(d^{4} k\right)^{\ell} e^{k a k+2 k r+R},
$$

where the polynomial operator means $P(k)=k_{1}^{\mu_{1}} \ldots k_{m}^{\mu_{m}}$. After doing the loop integration, the differential operators $\partial / \partial r_{i \mu}$ will give cause to outer momenta tensors. It may be done in effective way by using the identity

$$
\int_{0}^{\infty} d^{n} \alpha P\left(\frac{1}{2} \frac{\partial}{\partial r}\right) e^{-\frac{r^{2}}{a}}=\int_{0}^{\infty} d^{n} \alpha e^{-\frac{r^{2}}{a}} P\left(\frac{1}{2} \frac{\partial}{\partial r}-\frac{r}{a}\right)
$$

The calculation of the polynomial $P\left(\frac{1}{2} \frac{\partial}{\partial r}-\frac{r}{a}\right)$ can be automized by using the commutator $\left[\frac{\partial}{\partial r_{i}^{\mu}}, r_{j}^{v}\right]=$ $\delta_{i j} g^{\mu \nu}$. We have written a FORM [32] program that achieves the necessary commutations of the differential operators in a very efficient way.

The last point which remains to be discussed is the infrared cut-off we impose on the integration over the Fock-Schwinger parameters. This integration is multidimensional with the limits from 0 to $+\infty$. In order to arrive to a single cut-off parameter, we first transform the integral over an infinite space into an integral over a simplex convoluted with only one-dimensional improper integral. For that purpose, we use the $\delta$-function form of the identity

$$
1=\int_{0}^{\infty} d t \delta\left(t-\sum_{i=1}^{n} \alpha_{i}\right), \quad\left(\forall \alpha_{i} \geq 0\right),
$$


from which follows

$$
\Pi=\int_{0}^{\infty} d t t^{n-1} \int_{0}^{1} d^{n} \alpha \delta\left(1-\sum_{i=1}^{n} \alpha_{i}\right) \times W\left(t \alpha_{1}, \ldots, t \alpha_{1}\right),
$$

where $W$ represents the integrand of Schwinger parameters. The cut-off $\lambda$ is then introduced in a natural way:

$$
\int_{0}^{\infty} d t t^{n-1} \ldots \rightarrow \int_{0}^{1 / \lambda^{2}} d t t^{n-1} \ldots
$$

Such a cut-off makes the integral to be an analytic function without any singularities. In this way, all potential thresholds in the quark loop diagrams are removed together with corresponding branch points [31]. Within the covariant quark model, the cut-off parameter is universal for all processes, and its value, as obtained from a fit to data, is

$$
\lambda_{\text {cut }- \text { off }}=0.181 \mathrm{GeV} \text {. }
$$

The numerical evaluations have been done by a numerical program written in the fortran code.

As an example, let us consider a scalar one-loop two-point function:

$$
\Pi_{2}\left(p^{2}\right)=\int \frac{d^{4} k_{E}}{\pi^{2}} \frac{e^{-s k_{E}^{2}}}{\left[m^{2}+\left(k_{E}+\frac{1}{2} p_{E}\right)^{2}\right]\left[m^{2}+\left(k_{E}-\frac{1}{2} p_{E}\right)^{2}\right]},
$$

where the numerator factor $e^{-s k_{E}^{2}}$ comes from the product of nonlocal vertex form factors of Gaussian form. $k_{E}, p_{E}$ are Euclidean momenta $\left(p_{E}^{2}=-p^{2}\right)$. Doing the loop integration, one obtains

$$
\Pi_{2}\left(p^{2}\right)=\int_{0}^{\infty} d t \frac{t}{(s+t)^{2}} \int_{0}^{1} d \alpha \exp \left\{-t\left[m^{2}-\alpha(1-\alpha) p^{2}\right]+\frac{s t}{s+t}\left(\alpha-\frac{1}{2}\right)^{2} p^{2}\right\} .
$$

The function $\Pi_{2}\left(p^{2}\right)$ has a branch point at $p^{2}=4 m^{2}$, which occurs at $\alpha=1 / 2$. By introducing a cut-off in the $t$-integration, one obtains

$$
\Pi_{2}^{c}\left(p^{2}\right)=\int_{0}^{1 / \lambda^{2}} d t \frac{t}{(s+t)^{2}} \int_{0}^{1} d \alpha \exp \left\{-t\left[m^{2}-\alpha(1-\alpha) p^{2}\right]+\frac{s t}{s+t}\left(\alpha-\frac{1}{2}\right)^{2} p^{2}\right\},
$$

where the one-loop two-point function $\Pi_{2}^{c}\left(p^{2}\right)$ no longer has a branch point at $p^{2}=4 m^{2}$. The confinement scenario also allows us to include all possible, both two-quark and multi-quark, resonance states in our calculations.

\section{Some Nonleptonic Decays of Doubly Charmed Baryons}

We will consider the decays that belong to the same topology class:

$$
\begin{array}{llr}
\Xi_{c c}^{++} & \rightarrow \Xi_{c}^{+}\left(\Xi_{c}^{\prime+}\right)+\pi^{+}\left(\rho^{+}\right) & \text {T-Ia and W-IIb } \\
\Omega_{c c}^{+} & \rightarrow \Xi_{c}^{+}\left(\Xi_{c}^{\prime+}\right)+\bar{K}^{0}\left(K^{* 0}\right) & \text { T-Ib and W-IIb. }
\end{array}
$$

Their quantum numbers and interpolating currents are shown in Table 6. 
Table 6. Interpolating currents.

\begin{tabular}{llll}
\hline Baryon & $\boldsymbol{J}^{\boldsymbol{P}}$ & Interpolating Current & Mass (MeV) \\
\hline$\Xi_{c c}^{++}$ & $\frac{1}{2}^{+}$ & $\varepsilon_{a b c} \gamma^{\mu} \gamma_{5} u^{a}\left(c^{b} C \gamma_{\mu} c^{c}\right)$ & 3620.6 \\
$\Omega_{c c}^{+}$ & $\frac{1}{2}^{+}$ & $\varepsilon_{a b c} \gamma^{\mu} \gamma_{5} s^{a}\left(c^{b} C \gamma_{\mu} c^{c}\right)$ & 3710.0 \\
\hline$\Xi_{c}^{+}$ & $\frac{1}{2}^{+}$ & $\varepsilon_{a b c} \gamma^{\mu} \gamma_{5} c^{a}\left(u^{b} C \gamma_{\mu} s^{c}\right)$ & 2577.4 \\
$\Xi_{c}^{+}$ & $\frac{1}{2}^{+}$ & $\varepsilon_{a b c} c^{a}\left(u^{b} C \gamma_{5} s^{c}\right)$ & 2467.9 \\
\hline
\end{tabular}

The $W$-exchange contributions to the above decays fall into two classes:

1. The decays with a $\Xi_{c}^{\prime+}$-baryon containing a symmetric $\{u s\}$ diquark described by the interpolating current $\varepsilon_{a b c}\left(u^{b} C \gamma_{\mu} s^{c}\right)$. The $W$-exchange contribution is strongly suppressed due to the Körner-Pati-Woo (KPW) theorem [33,34], which states that the contraction of the flavor antisymmetric current-current operator with a flavor symmetric final state configuration is zero in the $S U(3)$ limit.

2. The decays with a $\Xi_{c}^{+}$-baryon containing a antisymmetric $[u s]$ diquark described by the interpolating current $\varepsilon_{a b c}\left(u^{b} C \gamma_{5} s^{c}\right)$. In this case, the $W$-exchange contribution is not a priori suppressed.

In our approach, the tree and $W$-exchange contributions are described by the Feynman diagrams shown in Figure 6.

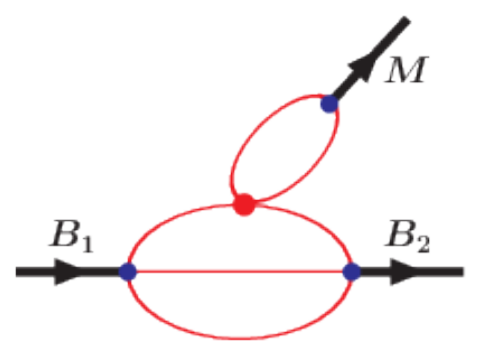

tree diagrams Ia, Ib

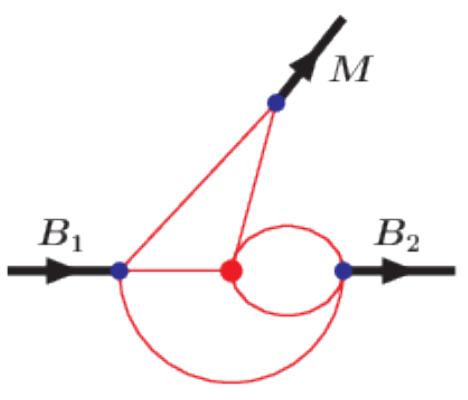

W-exchange diagram $\Pi b$

Figure 6. Diagrams describing the nonleptonic decay $B_{1} \rightarrow B_{2}+M$.

The matrix element is written down as

$$
<B_{2} M\left|\mathcal{H}_{\mathrm{eff}}\right| B_{1}>=\frac{G_{F}}{\sqrt{2}} V_{c s} V_{u d}^{\dagger} \bar{u}\left(p_{2}\right)\left(12 C_{T} M_{T}+12\left(C_{1}-C_{2}\right) M_{W}\right) u\left(p_{1}\right),
$$

where the combinations of the Wilson coefficients are given by

$$
C_{T}= \begin{cases}C_{T}=+\left(C_{2}+\xi C_{1}\right) & \text { charged meson } \\ C_{T}=-\left(C_{1}+\xi C_{2}\right) & \text { neutral meson. }\end{cases}
$$

The factor of $\xi=1 / N_{c}$ is set to zero in the numerical calculations. The contribution from the tree diagram factorizes into two pieces:

$$
M_{T}=M_{T}^{(1)} \cdot M_{T}^{(2)},
$$


where

$$
\begin{aligned}
M_{T}^{(1)} & =N_{c} g_{M} \int \frac{d^{4} k}{(2 \pi)^{4} i} \widetilde{\Phi}_{M}\left(-k^{2}\right) \operatorname{tr}\left(O_{L} S_{d}\left(k-w_{d} q\right) \Gamma_{M} S_{s(u)}\left(k+w_{s(u)} q\right)\right), \\
M_{T}^{(2)} & =g_{B_{1}} g_{B_{2}} \int \frac{d^{4} k_{1}}{(2 \pi)^{4} i} \int \frac{d^{4} k_{2}}{(2 \pi)^{4} i} \widetilde{\Phi}_{B_{1}}\left(-\vec{\Omega}_{1}^{2}\right) \widetilde{\Phi}_{B_{2}}\left(-\vec{\Omega}_{2}^{2}\right) \\
& \times \Gamma_{1} S_{c}\left(k_{2}\right) \gamma^{\mu} S_{c}\left(k_{1}-p_{1}\right) O_{R} S_{u(s)}\left(k_{1}-p_{2}\right) \widetilde{\Gamma}_{2} S_{s(u)}\left(k_{1}-k_{2}\right) \gamma_{\mu} \gamma_{5} .
\end{aligned}
$$

The $M_{T}^{(1)}$ is related to the leptonic decay constants:

$$
M_{T}^{(1)}=\left\{\begin{array}{lr}
-f_{P} \cdot q & \text { pseudoscalar meson } \\
+f_{V} m_{V} \cdot \epsilon_{V} & \text { vector meson. }
\end{array}\right.
$$

The semileptonic and nonleptonic two-body decays of the doubly charmed baryons $\Xi_{c c}^{++}, \Xi_{c c}^{+}$and $\Omega_{c c}^{+}$ have been studied in Reference [35] by considering those nonleptonic decay channels in which the decay proceeds solely via the factorizing contributions.

The $\mathrm{W}$-exchange contribution has no factorization and is written as genuine three-loop diagram:

$$
\begin{aligned}
M_{W} & =g_{B_{1}} g_{B_{2}} g_{M} \int \frac{d^{4} k_{1}}{(2 \pi)^{4} i} \int \frac{d^{4} k_{2}}{(2 \pi)^{4} i} \int \frac{d^{4} k_{3}}{(2 \pi)^{4} i} \widetilde{\Phi}_{B_{1}}\left(-\vec{\Omega}_{1}^{2}\right) \widetilde{\Phi}_{B_{2}}\left(-\vec{\Omega}_{2}^{2}\right) \widetilde{\Phi}_{M}\left(-P^{2}\right) \\
& \times 2 \Gamma_{1} S_{c}\left(k_{1}\right) \gamma^{\mu} S_{c}\left(k_{2}\right)\left(1-\gamma_{5}\right) S_{d}\left(k_{2}-k_{1}+p_{2}\right) \Gamma_{M} S_{s(u)}\left(k_{2}-k_{1}+p_{1}\right) \gamma_{\mu} \gamma_{5} \\
& \times \operatorname{tr}\left(S_{u(s)}\left(k_{3}\right) \widetilde{\Gamma}_{2} S_{s(u)}\left(k_{3}-k_{1}+p_{2}\right)\left(1+\gamma_{5}\right)\right) .
\end{aligned}
$$

Here, $\Gamma_{1} \otimes \widetilde{\Gamma}_{2}=I \otimes \gamma_{5}$ for $B_{2}=\Xi_{c}^{+}$, and $-\gamma_{v} \gamma_{5} \otimes \gamma^{v}$ for $B_{2}=\Xi_{c}^{\prime+}$. To verify the KPW theorem in the case of $B_{2}=\Xi_{c}^{\prime+}$, we use the identity

$$
\operatorname{tr}\left(S_{u}\left(k_{3}\right) \gamma_{v} S_{s}\left(k_{3}-k_{1}+p_{2}\right)\right)=-\operatorname{tr}\left(S_{s}\left(-k_{3}+k_{1}-p_{2}\right) \gamma_{v} S_{u}\left(-k_{3}\right)\right) .
$$

Then, by shifting $k_{3} \rightarrow-k_{3}+k_{1}-p_{2}$, one gets the same expression with opposite sign and $u \leftrightarrow s$ interchange. Thus, if $m_{u}=m_{s}$, then $M_{W} \equiv 0$. It directly confirms the KPW-theorem.

\section{Numerical Results}

The transition amplitudes in terms of invariant amplitudes are written down as

$$
\begin{aligned}
<B_{2} P\left|\mathcal{H}_{\mathrm{eff}}\right| B_{1}> & =\frac{G_{F}}{\sqrt{2}} V_{c s}^{*} V_{u d} \bar{u}\left(p_{2}\right)\left(A+\gamma_{5} B\right) u\left(p_{1}\right) \\
<B_{2} V\left|\mathcal{H}_{\mathrm{eff}}\right| B_{1}> & =\frac{G_{F}}{\sqrt{2}} V_{c s}^{*} V_{u d} \\
& \times \bar{u}\left(p_{2}\right) \epsilon_{V \delta}^{*}\left(\gamma^{\delta} V_{\gamma}+p_{1}^{\delta} V_{p}+\gamma_{5} \gamma^{\delta} V_{5 \gamma}+\gamma_{5} p_{1}^{\delta} V_{5 p}\right) u\left(p_{1}\right)
\end{aligned}
$$

The invariant amplitudes can be expressed in terms of helicity amplitudes as

$$
\begin{array}{lll}
H_{\frac{1}{2} t}^{V}=\sqrt{Q_{+}} A \quad H_{\frac{1}{2} t}^{A}=\sqrt{Q_{-}} B & \\
H_{\frac{1}{2} 0}^{V}=+\sqrt{Q_{-} / q^{2}}\left(m_{+} V_{\gamma}+\frac{1}{2} Q_{+} V_{p}\right) & H_{\frac{1}{2} 1}^{V}=-\sqrt{2 Q_{-}} V_{\gamma} \\
H_{\frac{1}{2} 0}^{A}=+\sqrt{Q_{+} / q^{2}}\left(m_{-} V_{5 \gamma}+\frac{1}{2} Q_{-} V_{5 p}\right) & H_{\frac{1}{2} 1}^{A}=-\sqrt{2 Q_{+}} V_{5 \gamma} .
\end{array}
$$


Here, $m_{ \pm}=m_{1} \pm m_{2}, Q_{ \pm}=m_{ \pm}^{2}-q^{2}$ and $\left|\mathbf{p}_{2}\right|=\lambda^{1 / 2}\left(m_{1}^{2}, m_{2}^{2}, q^{2}\right) /\left(2 m_{1}\right)$. The parity relations have taken place: $H_{-\lambda_{2},-\lambda_{M}}^{V}=+H_{\lambda_{2}, \lambda_{M}}^{V}, H_{-\lambda_{2},-\lambda_{M}}^{A}=-H_{\lambda_{2}, \lambda_{M}}^{A}$. Finally, the decay widths are written as

$$
\begin{aligned}
\Gamma\left(B_{1} \rightarrow B_{2}+P(V)\right) & =\frac{G_{F}^{2}}{32 \pi}\left|V_{c s}^{*} V_{u d}\right|^{2} \frac{\left|\mathbf{p}_{2}\right|}{m_{1}^{2}} \mathcal{H}_{P(V)} \\
\mathcal{H}_{P} & =\left|H_{\frac{1}{2} t}\right|^{2}+\left|H_{-\frac{1}{2} t}\right|^{2}, \\
\mathcal{H}_{V} & =\left|H_{\frac{1}{2} 0}\right|^{2}+\left|H_{-\frac{1}{2} 0}\right|^{2}+\left|H_{\frac{1}{2} 1}\right|^{2}+\left|H_{-\frac{1}{2}-1}\right|^{2},
\end{aligned}
$$

where $H=H^{V}-H^{A}$. All model parameters have been fixed in our previous studies, except for the size parameter $\Lambda_{c c}$ of the doubly charmed baryons. As a first approximation, we equate the size parameter of doubly charmed baryons with that of singly charmed baryons, i.e., we take $\Lambda_{c c}=\Lambda_{c}=0.8675 \mathrm{GeV}$, where we adopt the value of $\Lambda_{c}$ from Reference [36] obtained by fitting the magnetic moment of $\Lambda_{c}$ to its experimental value.

Numerical results for the helicity amplitudes and decay widths are displayed in the Tables 7-10.

Our results highlight the importance of the KPW theorem for the nonleptonic decays when the final state involves a $\Xi^{\prime+}$ baryon containing a symmetric $\{s u\}$ diquark. Tables $7-10$ show that the relevant $W$-exchange contributions are nonzero but are strongly suppressed. Nonzero values result from SU(3) breaking effects, which are accounted for in our approach. Take, for example, the decay $\Xi_{c c}^{++} \rightarrow \Xi_{c}^{\prime+}+\pi^{+}$. When compared to the tree contribution, the $S U(3)$ breaking effects amount to $\sim(2-4) \%$. While the consequences of the KPW theorem for the $W$-exchange contribution are also incorporated in the pole model approach of Reference [37] (see Figure 7), they are not included in the final-state interaction approach of Reference [38].
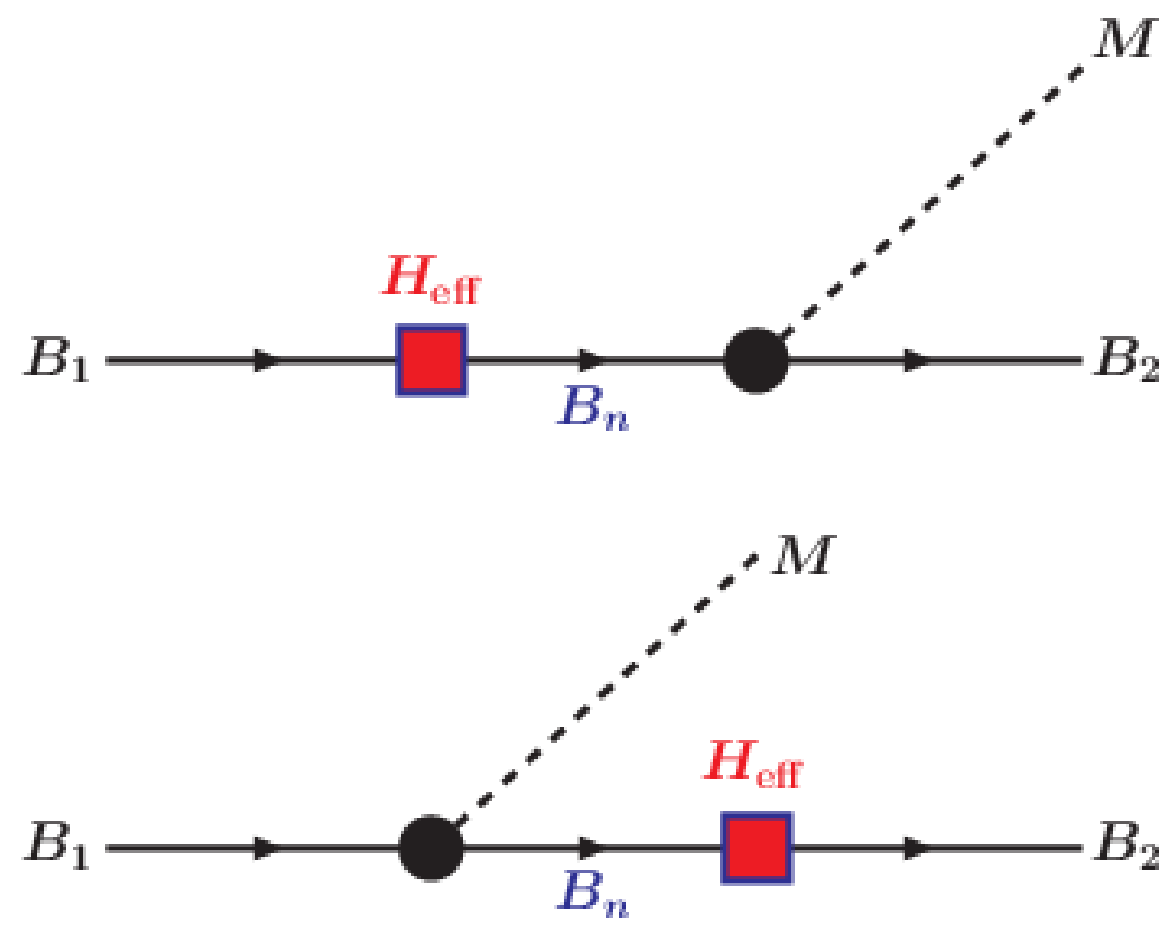

Figure 7. Schematic image of the pole model. 
Table 7. Decays $\Omega_{\mathcal{c}}^{+} \rightarrow \Xi_{c}^{\prime+}+\bar{K}^{0}\left(\bar{K}^{* 0}\right)$.

\begin{tabular}{cccc}
\hline Helicity & Tree Diagram & $\boldsymbol{W}$ Diagram & Total \\
\hline$H_{\frac{1}{2} t}^{V}$ & 0.20 & -0.01 & 0.19 \\
$H_{\frac{1}{2} t}^{A}$ & 0.25 & -0.01 & 0.24 \\
\hline \multicolumn{4}{c}{$\Gamma\left(\Omega_{c c}^{+} \rightarrow \Xi_{c}^{\prime+}+\bar{K}^{0}\right)=0.15 \cdot 10^{-13} \mathrm{GeV}$} \\
\hline$H_{\frac{1}{2} 0}^{V}$ & -0.25 & $0.04 \times 10^{-1}$ & -0.25 \\
$H_{\frac{1}{2}}^{A} 0$ & -0.50 & 0.01 & -0.49 \\
$H_{\frac{1}{2}}^{V} 1$ & 0.27 & -0.01 & 0.26 \\
$H_{\frac{1}{2}}^{A} 1$ & 0.56 & $0.04 \times 10^{-2}$ & 0.56 \\
\hline$\Gamma\left(\Omega_{c c}^{+} \rightarrow \Xi_{c}^{\prime+}+\bar{K}^{* 0}\right)=0.74 \cdot 10^{-13} \mathrm{GeV}$ \\
\hline \multicolumn{4}{c}{}
\end{tabular}

Table 8. Decays $\Omega_{c c}^{+} \rightarrow \Xi_{c}^{+}+\bar{K}^{0}\left(\bar{K}^{* 0}\right)$.

\begin{tabular}{|c|c|c|c|}
\hline Helicity & Tree Diagram & W Diagram & Total \\
\hline$H_{\frac{1}{2} t}^{V}$ & -0.35 & 1.06 & 0.71 \\
\hline$H_{\frac{1}{2} t}^{A}$ & -0.10 & 0.31 & 0.21 \\
\hline \multicolumn{4}{|c|}{$\Gamma\left(\Omega_{c c}^{+} \rightarrow \Xi_{c}^{+}+\bar{K}^{0}\right)=0.95 \cdot 10^{-13} \mathrm{GeV}$} \\
\hline$H_{\frac{1}{2} 0}^{V}$ & 0.50 & -0.69 & -0.19 \\
\hline$H_{\frac{1}{2} 0}^{A}$ & 0.18 & -0.45 & -0.27 \\
\hline$H_{\frac{1}{2} 1}^{V}$ & -0.11 & -0.24 & -0.35 \\
\hline$H_{\frac{1}{2} 1}^{A}$ & -0.18 & 0.66 & 0.48 \\
\hline \multicolumn{4}{|c|}{$\Gamma\left(\Omega_{c c}^{+} \rightarrow \Xi_{c}^{+}+\bar{K}^{* 0}\right)=0.62 \cdot 10^{-13} \mathrm{GeV}$} \\
\hline
\end{tabular}

Table 9. Decays $\Xi_{c c}^{++} \rightarrow \Xi_{c}^{\prime+}+\pi^{+}\left(\rho^{+}\right)$.

\begin{tabular}{cccc}
\hline Helicity & Tree Diagram & W Diagram & Total \\
\hline$H_{\frac{1}{2} t}^{V}$ & -0.38 & -0.01 & -0.39 \\
$H_{\frac{1}{2} t}^{A}$ & -0.55 & -0.02 & -0.57 \\
\hline \multicolumn{4}{c}{$\Gamma\left(\Xi_{c c}^{++} \rightarrow \Xi_{c}^{\prime+}+\pi^{+}\right)=0.82 \cdot 10^{-13} \mathrm{GeV}$} \\
\hline$H_{\frac{1}{2} 0}^{V}$ & 0.60 & $0.04 \times 10^{-1}$ & 0.61 \\
$H_{\frac{1}{2}}^{A} 0$ & 1.20 & 0.01 & 1.21 \\
$H_{\frac{1}{2}}^{V} 1$ & -0.49 & -0.01 & -0.50 \\
$H_{\frac{1}{2} 1}^{A}$ & -1.27 & $0.01 \times 10^{-1}$ & -1.27 \\
\hline \multicolumn{4}{c}{$\Gamma\left(\Xi_{c c}^{++} \rightarrow \Xi_{c}^{\prime+}+\rho^{+}\right)=4.27 \cdot 10^{-13} \mathrm{GeV}$} \\
\hline \multicolumn{4}{l}{}
\end{tabular}


Table 10. Decays $\Xi_{c c}^{++} \rightarrow \Xi_{c}^{+}+\pi^{+}\left(\rho^{+}\right)$

\begin{tabular}{cccc}
\hline Helicity & Tree Diagram & $\boldsymbol{W}$ Diagram & Total \\
\hline$H_{\frac{1}{2} t}^{V}$ & -0.70 & 0.99 & 0.29 \\
$H_{\frac{1}{2} t}^{A}$ & -0.21 & 0.30 & 0.09 \\
\hline \multicolumn{4}{c}{$\Gamma\left(\Xi_{c c}^{++} \rightarrow \Xi_{c}^{+}+\pi^{+}\right)=0.18 \cdot 10^{-13} \mathrm{GeV}$} \\
\hline$H_{\frac{1}{2} 0}^{V}$ & 1.17 & -0.70 & 0.47 \\
$H_{\frac{1}{2}}^{A} 0$ & 0.45 & -0.44 & 0.003 \\
$H_{\frac{1}{2}}^{V} 1$ & -0.20 & -0.23 & -0.43 \\
$H_{\frac{1}{2} 1}^{A}$ & -0.41 & 0.62 & 0.21 \\
\hline \multicolumn{4}{c}{$\Gamma\left(\Xi_{\mathcal{~}}^{++} \rightarrow \Xi_{c}^{+}+\rho^{+}\right)=0.63 \cdot 10^{-13} \mathrm{GeV}$} \\
\hline \multicolumn{4}{c}{}
\end{tabular}

In Table 11, we compare our rate results with the results of some other approaches [37-42]. All calculations approximately agree on the rate of the decay $\Xi_{c c}^{++} \rightarrow \Xi_{c}^{\prime+}+\rho^{+}$, which is predicted to have a large branching ratio of $\sim 16 \%$. In our calculation, this mode is predicted to have by far the largest branching ratio of the decays analyzed in this paper. As concerns the decay $\Xi_{c c}^{++} \rightarrow \Xi_{c}^{+}+\pi^{+}$ discovered by the LHCb Collaboration [15], we find a branching ratio of $\mathcal{B}\left(\Xi_{c c}^{++} \rightarrow \Xi_{c}^{+} \pi^{+}\right)=$ $0.70 \%$ using the central value of the life time measurement in Reference [14]. The small value of the branching ratio results from a substantial cancellation of the tree and $W$-exchange contributions. The branching ratio is somewhat smaller than the branching ratio $\mathcal{B}\left(\Xi_{c c}^{++} \rightarrow \Sigma_{c}^{++}+\bar{K}^{0}\right)=1.28 \%$ calculated in Reference [43]. We think that the latter mode is more dominant in comparison with $\Xi_{c c}^{++} \rightarrow \Xi_{c}^{+} \pi^{+}$. We predict a branching ratio considerably smaller than the range of branching fractions (6.66 - 15.79) \% calculated in Reference [37]. In our opinion, the calculations done in Reference [37] involve generous approximations for the errors, which are hard to quantify.

Table 11. Comparison with other approaches. Abbreviation: $M=N R Q M, T=H Q E T$.

\begin{tabular}{|c|c|c|c|c|c|c|}
\hline \multirow{2}{*}{ Mode } & \multicolumn{6}{|c|}{ Width (in $10^{-13} \mathrm{GeV}$ ) } \\
\hline & GIKLT [22,43] & DS $[37,39]$ & JHL [38] & WYZ [40] & YJLLWZ [41] & KL [42] \\
\hline$\Xi_{c c}^{++} \rightarrow \Sigma_{c}^{++}+\bar{K}^{0}$ & 0.33 & & & & & \\
\hline$\Xi_{c c}^{++} \rightarrow \Sigma_{c}^{++}+\bar{K}^{* 0}$ & 1.38 & & & & & \\
\hline$\Omega_{c c}^{+} \rightarrow \Xi_{c}^{\prime+}+\bar{K}^{0}$ & 0.15 & $\begin{array}{c}0.31(\mathrm{M}) \\
0.59(\mathrm{~T})\end{array}$ & & & & \\
\hline$\Omega_{c c}^{+} \rightarrow \Xi_{c}^{+}+\bar{K}^{0}$ & 0.95 & $\begin{array}{c}0.68(\mathrm{M}) \\
1.08(\mathrm{~T})\end{array}$ & & & & \\
\hline$\Omega_{c c}^{+} \rightarrow \Xi_{c}^{\prime+}+\bar{K}^{* 0}$ & 0.74 & & $2.64_{-1.79}^{+2.72}$ & & & \\
\hline$\Omega_{c c}^{+} \rightarrow \Xi_{c}^{+}+\bar{K}^{* 0}$ & 0.62 & & $1.38_{-0.95}^{+1.49}$ & & & \\
\hline$\Xi_{c c}^{++} \rightarrow \Xi_{c}^{\prime+}+\pi^{+}$ & 0.82 & $\begin{array}{l}1.40(\mathrm{M}) \\
1.93(\mathrm{~T})\end{array}$ & & 1.10 & & \\
\hline$\Xi_{c c}^{++} \rightarrow \Xi_{c}^{+}+\pi^{+}$ & 0.18 & $\begin{array}{l}1.71(\mathrm{M}) \\
2.39(\mathrm{~T})\end{array}$ & & 1.57 & 1.58 & 2.25 \\
\hline$\Xi_{c c}^{++} \rightarrow \Xi_{c}^{\prime+}+\rho^{+}$ & 4.27 & & $4.25_{-0.19}^{+0.32}$ & 4.12 & 3.82 & \\
\hline$\Xi_{c c}^{++} \rightarrow \Xi_{c}^{+}+\rho^{+}$ & 0.63 & & $4.11_{-0.86}^{+1.37}$ & 3.03 & 2.76 & 6.70 \\
\hline
\end{tabular}


The only free parameter in our approach is the size parameter $\Lambda_{c c}$ of the double heavy baryons, for which we have chosen $\Lambda_{c c}=0.8675 \mathrm{GeV}$ in Tables 7-10. In order to estimate the uncertainty caused by the choice of the size parameter, we allow the size parameter to vary from 0.6 to $1.135 \mathrm{GeV}$. We evaluate the mean $\bar{\Gamma}=\sum \Gamma_{i} / N$ and the mean square deviation $\sigma^{2}=\sum\left(\Gamma_{i}-\bar{\Gamma}\right)^{2} / N$. The results for $N=5$ are shown in Table 12. The rate errors amount to $6-15 \%$. Since the dependence of the rates on $\Lambda_{c c}$ is nonlinear, the central values of the rates in Table 12 do not agree with the rate values in Tables $7-10$.

Table 12. Estimating uncertainties in the decay widths.

\begin{tabular}{lc}
\hline \multicolumn{1}{c}{ Mode } & Width (in 10 $\mathbf{~}^{\mathbf{1 3}} \mathbf{G e V}$ ) \\
\hline$\Omega_{c c}^{+} \rightarrow \Xi_{c}^{\prime+}+\bar{K}^{0}$ & $0.14 \pm 0.01$ \\
$\Omega_{c c}^{+} \rightarrow \Xi_{c}^{\prime+}+\bar{K}^{* 0}$ & $0.72 \pm 0.06$ \\
\hline$\Omega_{c c}^{+} \rightarrow \Xi_{c}^{+}+\bar{K}^{0}$ & $0.87 \pm 0.13$ \\
$\Omega_{c c}^{+} \rightarrow \Xi_{c}^{+}+\bar{K}^{* 0}$ & $0.58 \pm 0.07$ \\
\hline$\Xi_{c c}^{++} \rightarrow \Xi_{c}^{\prime+}+\pi^{+}$ & $0.77 \pm 0.05$ \\
$\Xi_{c c}^{++} \rightarrow \Xi_{c}^{\prime+}+\rho^{+}$ & $4.08 \pm 0.29$ \\
\hline$\Xi_{c c}^{++} \rightarrow \Xi_{c}^{+}+\pi^{+}$ & $0.16 \pm 0.02$ \\
$\Xi_{c c}^{++} \rightarrow \Xi_{c}^{+}+\rho^{+}$ & $0.59 \pm 0.04$ \\
\hline
\end{tabular}

\section{Outlook}

The discovery of the doubly charmed baryon $\Xi_{c c}^{++}$by the LHCb Collaboration $[13,14]$ and the first observation of its two-body nonleptonic decay $\Xi_{c c}^{++} \rightarrow \Xi_{c}^{+}+\pi^{+}$[15] provided strong encouragement for further theoretical analysis of the weak decays of doubly charmed baryons. As well known, such decays are described by the quark diagrams, which have several different topologies. One class of the diagrams having the so-called factorizing topology is easy to evaluate in almost model independent way, whereas another class with the internal $W$-exchange is extremely difficult to handle theoretically. In this lecture, we gave a basic introduction to the methods of how to calculate the relevant diagrams with any topologies in self-consistent way on the same basis. The theoretical background used for this purpose is the covariant constituent model previously developed in our papers. As the first step, we concentrated on the description of Cabibbo-favored nonleptonic two-body decays of the doubly charmed ground state baryons $\Xi_{c c}^{++}$and $\Omega_{c c}^{+}$where we have limited our analysis to the $\frac{1}{2} \rightarrow \frac{1}{2}+P(V)$ decay channels. It would be straightforward to also include $\frac{1}{2} \rightarrow \frac{3}{2}+P(V)$ the nonleptonic decays in the future. In addition, the study could be extended to the description of singly and doubly suppressed Cabibbo decays, not only for doubly charmed baryon decays but also for singly charmed baryon decays.

Funding: This research was partly supported by the PRISMA+Cluster of Excellence (Mainz Uni.).

Acknowledgments: I would like to thank Thomas Gutsche, Jürgen Körner, Valery Lyubovitskij, Pietro Santorelli and Zhomart Tyulemissov for their collaboration in which the results discussed in this lecture have been obtained.

Conflicts of Interest: The author declares no conflict of interest.

\section{References}

1. Gell-Mann, M. A Schematic Model of Baryons and Mesons. Phys. Lett. 1964, 8, 214-215. [CrossRef]

2. Zweig, G. An SU(3) Model for Strong Interaction Symmetry and Its Breaking, Version 1; PREPRINT CERN-TH-401; 1964; pp. 1-26. Available online: inspirehep.net/record/11881/files/CM-P00042883.pdf (accessed on 6 January 2020). 
3. Zweig, G. An SU(3) Model for Strong Interaction Symmetry and Its Breaking, Version 2; PREPRINT CERN-TH-412; 1964; pp. 1-68. Available online: https://cds.cern.ch/record/570209/files/CERN-TH412.pdf (accessed on 6 January 2020).

4. Bjorken, J.D.; Glashow, S.L. Elementary Particles and SU(4). Phys. Lett. 1964, 11, 255-257. [CrossRef]

5. Glashow, S.L.; Iliopoulos, J.; Maiani, L. Weak Interactions with Lepton-Hadron Symmetry. Phys. Rev. D 1970, 2, 1285-1292. [CrossRef]

6. De Rujula, A.; Georgi, H.; Glashow, S.L. Hadron Masses in a Gauge Theory. Phys. Rev. D 1975, 12, 147-162. [CrossRef]

7. Körner, J.G.; Kramer, M.; Pirjol, D. Heavy baryons. Prog. Part. Nucl. Phys. 1994, 33, 787-868. [CrossRef]

8. Tanabashi, M.; Hagiwara, K.; Hikasa, K.; Nakamura, K.; Sumino, Y.; Takahashi, F.; Tanaka, J.; Agashe, K.; Aielli, G.; Amsler, C.; et al. Review of particle physics. Phys. Rev. D 2018, 98, 030001. [CrossRef]

9. Ablikim, M.; Achasov, M.N.; Ai, X.C.; Albayrak, O.; Albrecht, M.; Ambrose, D.J.; Amoroso, A.; An, F.F.; An, Q.; Bai, J.Z.; et al. Measurements of absolute hadronic branching fractions of $\Lambda_{c}^{+}$baryon. Phys. Rev. Lett. 2016, 116, 052001. [CrossRef]

10. Li, Y.B.; Shen, C.P.; Yuan, C.Z.; Adachi, I.; Aihara, H.; Al Said, S.; Asner, D.M.; Aushev, T.; Ayad, R.; Badhrees, I.; et al. First, measurements of absolute branching fractions of the $\Xi_{c}^{0}$ baryon at Belle. Phys. Rev. Lett. 2019, 122, 082001. [CrossRef]

11. Li, Y.B.; Shen, C.P.; Adachi, I.; Ahn, J.K.; Aihara, H.; Al Said, S.; Asner, D. M.; Atmacan, H.; Aushev, T.; Ayad, R.; et al. First measurements of absolute branching fractions of the $\Xi_{c}^{+}$baryon at Belle. Phys. Rev. D 2019, 100, no. 3, 031101. [CrossRef]

12. Ocherashvili, A.; Moinester, M.A.; Russ, J.; Engelfried, J.; Torres, I.; Akgun, U.; Alkhazov, G.; Amaro-Reyes, J.; Atamantchouk, A.G.; Ayan, A.S.; et al. Confirmation of the double charm baryon $\Xi_{c c}^{+}(3520)$ via its decay $p D^{+} K^{-}$. Phys. Lett. B 2005, 628, 18. [CrossRef]

13. Aaij, R.; Adeva, B.; Adinolfi, M.; Ajaltouni, Z.; Akar, S.; Albrecht, J.; Alessio, F.; Alexander, M.; Albero, A.A.; Ali, S.; et al. Observation of the doubly charmed baryon $\Xi_{c c}^{++}$. Phys. Rev. Lett. 2017, 119, 112001. [CrossRef] [PubMed]

14. Aaij, R.; Adeva, B.; Adinolfi, M.; Aidala, C.A.; Ajaltouni, Z.; Akar, S.; Albicocco, P.; Albrecht, J.; Alessio, F.; Alexander, M.; et al. Measurement of the lifetime of the doubly charmed baryon $\Xi_{c c}^{++}$. Phys. Rev. Lett. 2018, 121, 052002. [CrossRef] [PubMed]

15. Aaij, R.; Adeva, B; Adinolfi, M.; Aidala, C.A.; Ajaltouni, Z.; Akar, S.; Albicocco, P.; Albrecht, J.; Alessio, F.; Alexander, M.; et al. First, observation of the doubly charmed baryon decay $\Xi_{c c}^{++} \rightarrow \Xi_{c}^{+} \pi^{+}$. Phys. Rev. Lett. 2018, 121, 162002. [CrossRef] [PubMed]

16. Körner, J.G.; Krämer, M. Exclusive nonleptonic charm baryon decays. Z. Phys. C 1992, 55, 659. [CrossRef]

17. De Rujula, A.; Georgi, H.; Glashow, S.L. Vector Model of the Weak Interactions. Phys. Rev. D 1975, $12,3589$. [CrossRef]

18. Ebert, D.; Faustov, R.N.; Galkin, V.O.; Martynenko, A.P. Mass spectra of doubly heavy baryons in the relativistic quark model. Phys. Rev. D 2002, 66, 014008. [CrossRef]

19. Fleck, S.; Richard, J.M. Baryons with double charm. Prog. Theor. Phys. 1989, 82, 760. [CrossRef]

20. Karliner, M.; Rosner, J.L. Baryons with two heavy quarks: Masses, production, decays, and detection. Phys. Rev. D 2014, 90, 094007. [CrossRef]

21. Gutsche, T.; Ivanov, M.A.; Körner, J.G.; Lyubovitskij, V.E. Novel ideas in nonleptonic decays of double heavy baryons. Particles 2019, 2, 339-356. [CrossRef]

22. Gutsche, T.; Ivanov, M.A.; Körner, J.G.; Lyubovitskij, V.E.; Tyulemissov, Z. Ab initio three-loop calculation of the $W$-exchange contribution to nonleptonic decays of double charm baryons. Phys. Rev. D 2019, 99, 056013 [CrossRef]

23. Fayyazuddin and Riazuddin. A Modern Introduction to Particle Physics; World Scientific: Singapore, 1994; 656p.

24. Efimov, G.V.; Ivanov, M.A.; Lyubovitskij, V.E. Quark - diquark approximation of the three quark structure of baryons in the quark confinement model. Z. Phys. C 1990, 47, 583-594. [CrossRef]

25. Ioffe, B.L. On The Choice Of Quark Currents In The Qcd Sum Rules For Baryon Masses. Z. Phys. C 1983, 18, 67. [CrossRef]

26. Reinders, L.J.; Rubinstein, H.; Yazaki, S. Hadron Properties from QCD Sum Rules. Phys. Rept. 1985, $127,1$. [CrossRef] 
27. Leibovich, A.K.; Ligeti, Z.; Stewart, I.W.; Wise, M.B.; Predictions for nonleptonic Lambda(b) and Theta(b) decays, Phys. Lett. B 2004, 586, 337. [CrossRef]

28. Ivanov, M.A.; Khomutenko, O.E.; Mizutani, T. Form-factors of semileptonic decays of heavy mesons in the quark confinement model. Phys. Rev. D 1992, 46, 3817. [CrossRef]

29. Ivanov, M.A.; Kalinovsky, Y.L.; Roberts, C.D. Survey of heavy meson observables. Phys. Rev. D 1999, 60, 034018. [CrossRef]

30. Ivanov, M.A.; Körner, J.G.; Tran, C.T. Exclusive decays $B \rightarrow \ell^{-} \bar{v}$ and $B \rightarrow D^{(*)} \ell^{-} \bar{v}$ in the covariant quark model. Phys. Rev. D 2015, 92, 114022 [CrossRef]

31. Branz, T.; Faessler, A.; Gutsche, T.; Ivanov, M.A.; Körner, J.G.; Lyubovitskij, V.E. Relativistic constituent quark model with infrared confinement. Phys. Rev. D 2010, 81, 034010. [CrossRef]

32. Vermaseren, J.A.M. The FORM project. Nucl. Phys. Proc. Suppl. 2008, 183, 19. [CrossRef]

33. Körner, J.G. Octet behaviour of single-particle matrix elements $<B^{\prime}|H(W)| B>$ and $\left\langle M^{\prime}|H(W)| M>\right.$ using a weak current current quark Hamiltonian. Nucl. Phys. B 1971, 25, 282.

34. Pati, J.C.; Woo, C.H. Delta $I=1 / 2$ rule with fermion quarks. Phys. Rev. D 1971, 3, 2920. [CrossRef]

35. Gutsche, T.; Ivanov, M.A.; Körner, J.G. Lyubovitskij, V.E.; Tyulemissov, Z. Analysis of the semileptonic and nonleptonic two-body decays of the double heavy charm baryon states $\Xi_{c c}^{++}, \Xi_{c c}^{+}$and $\Omega_{c c}^{+}$. Phys. Rev. D 2019, 100, 114037. [CrossRef]

36. Gutsche, T.; Ivanov, M.A.; Körner, J.G.; Lyubovitskij, V.E.; Santorelli, P. Semileptonic decays $\Lambda_{c}^{+} \rightarrow$ $\Lambda \ell^{+} v_{\ell}(\ell=e, \mu)$ in the covariant quark model and comparison with the new absolute branching fraction measurements of Belle and BESIII. Phys. Rev. D 2016, 93, 034008. [CrossRef]

37. Sharma, N.; Dhir, R. Estimates of $W$-exchange contributions to $\Xi_{c c}$ decays. Phys. Rev. D 2017, $96,113006$. [CrossRef]

38. Jiang, L.J.; He, B.; Li, R.H. Weak Decays of Doubly Heavy Baryons: $\mathcal{B}_{c c} \rightarrow \mathcal{B}_{c}$ V. Eur. Phys. J. C 2018, 78, 961. [CrossRef]

39. Dhir, R.; Sharma, N. Weak decays of doubly heavy charm $\Omega_{c c}^{+}$baryon. Eur. Phys. J. C 2018, 78, 743. [CrossRef]

40. Wang, W.; Yu, F.S.; Zhao, Z.X. Weak decays of doubly heavy baryons: The $1 / 2 \rightarrow 1 / 2$ case. Eur. Phys. J. C 2017, 77, 781. [CrossRef]

41. Yu, F. S.; Jiang, H.Y.; Li, R.H.; Lü, C.D.; Wang, W.; Zhao, Z.X. Discovery Potentials of Doubly Charmed Baryons. Chin. Phys. C 2018, 42, 051001. [CrossRef]

42. Kiselev, V.V.; Likhoded, A.K. Baryons with two heavy quarks. Phys. Usp. 2002, 45, 455. [CrossRef]

43. Gutsche, T.; Ivanov, M.A.; Körner, J.G.; Lyubovitskij, V.E. Decay chain information on the newly discovered double charm baryon state $\Xi_{c c}^{++}$. Phys. Rev. D 2017, 96, 054013. [CrossRef]

(C) 2020 by the author. Licensee MDPI, Basel, Switzerland. This article is an open access article distributed under the terms and conditions of the Creative Commons Attribution (CC BY) license (http:// creativecommons.org/licenses/by/4.0/). 